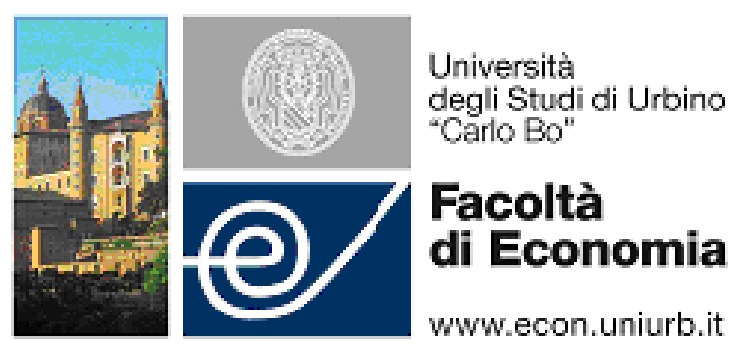

WP-EMS

Working Papers Series in Economics, Mathematics and Statistics

"On the complicated price dynamics of a simple one-dimensional discontinuous financial market model with heterogeneous interacting traders"

- Fabio Tramontana, (U. Ancona)

- Frank Westerhoff (U. Bamberg)

- Laura Gardini (U. Urbino) 


\title{
On the complicated price dynamics of a simple one-dimensional discontinuous financial market model with heterogeneous interacting traders
}

\author{
${ }^{1}$ Fabio Tramontana, ${ }^{2}$ Frank Westerhoff, ${ }^{3}$ Laura Gardini \\ ${ }^{1}$ Università Politecnica delle Marche \\ ${ }^{2}$ University of Bamberg \\ ${ }^{3}$ Università degli Studi di Urbino
}

\begin{abstract}
We develop a financial market model with heterogeneous interacting agents: market makers adjust prices with respect to excess demand, chartists believe in the persistence of bull and bear markets and fundamentalists bet on mean reversion. Moreover, speculators trade asymmetrically in over- and undervalued markets and while some of them determine the size of their orders via linear trading rules others always trade the same amount of assets. The dynamics of our model is driven by a one-dimensional discontinuous map. Despite the simplicity of our model, analytical, graphical and numerical analysis reveals a surprisingly rich set of interesting dynamical behaviors.
\end{abstract}

Key words

Financial markets; heterogeneous agents; technical and fundamental analysis; nonlinear dynamics; discontinuous map, bifurcation analysis.

JEL classification

D84, G12, G14, G15 


\section{Introduction}

Spectacular financial market bubbles have repeatedly been observed in the past, often followed by equally stunning crashes. In some cases, these events even had an impact on the real economy, triggering deeper recessions, for instance. Moreover, the volatility in financial markets may be regarded as excessively high in the sense that prices fluctuate more strongly than warranted by the underlying fundamentals. Also extreme price changes, which make up a large part of financial market risk, occur quite frequently. Detailed empirical accounts on these intriguing phenomena are provided by Sornette (2003), Shiller (2005, 2008) and Lux (2009b).

Obviously, it is important to understand what drives the dynamics of financial markets. Bouchaud et al. (2009) present significant empirical evidence showing that asset prices mainly adjust with respect to the markets' order imbalances which, of course, originate from the transactions of its market participants. Fortunately, we at least have some empirical evidence on how agents determine their speculative orders. As can be seen from empirical studies involving questionnaires (summarized by Menkoff and Taylor 2007), market participants rely on both technical and fundamental trading rules to determine the course of the market. Technical analysis is a trading method that seeks to identify trading signals from past price movements (Murphy 1999). As a result, technicians - also called chartists - may have a destabilizing effect on the dynamics of financial markets. Fundamental analysis presumes that prices will mean-revert toward fundamental values (Graham and Dodd 1951), generally inducing some kind of market stability. Similar insights are obtained in laboratory experiments in which human subjects trade in a controlled financial market environment (Smith et al. 1988, Hommes et al. 2005).

But how exactly do markets with a diverse ecology of interacting technical and fundamental traders function? Models with heterogeneous agents take exactly this issue into account. For recent surveys of this burgeoning field of research, see Chiarella et al. (2009), Hommes and Wagener (2009), Lux (2009a) and Westerhoff (2009), among others. While some stochastic versions of these models aim at matching the stylized facts of financial markets - several interesting contributions are presented in LeBaron (2006), Lux (2009b) and Chen et al. (2009) ${ }^{1}{ }_{-}^{-}$ other studies focus on deterministic setups to improve our basic knowledge of what drives prices in financial markets.

\footnotetext{
${ }^{1}$ For specific examples see Gaunersdorfer and Hommes (2007), He and Li (2008), Franke (2009) and Franke and Westerhoff (2009).
} 
Let us briefly outline a few of these frameworks in order to appreciate the insights made in this exciting field of research - and to clarify the extent to which our model differs from previous works in this field.

- One interesting finding is due to Day and Huang (1990), who show that endogenous price dynamics may be triggered by nonlinear trading rules. In their model, chartists apply a linear trading rule, and their orders destabilize the market close to the fundamental value. The trading behavior of fundamentalists is nonlinear. The more the price deviates from the fundamental value, the more aggressive they become. Eventually, orders placed by fundamentalists exceed orders placed by chartists, and prices are pushed back towards fundamental values. However, close to the fundamental value, chartists again dominate the market and the process repeats itself, albeit in an intricate, unpredictable way. Related models featuring nonlinear technical trading rules have been elaborated by Chiarella (1992), Chiarella et al. (2002), and others.

- Another interesting insight is that when agents switch between technical and fundamental analysis, a similar dynamic behavior can emerge. Let us suppose the market is dominated by destabilizing chartists. In this case, it is likely that prices disconnect from fundamentals. However, when fundamental analysis becomes more popular, a period of price stability, together with a convergence towards fundamental values, may set in. Brock and Hommes (1998) develop a model in which agents switch between trading rules with respect to their past performance and thus display some kind of learning behavior. In Kirman (1991), agents have social interactions that may lead to swings of opinion. In Lux (1998), traders compare the performance of trading rules but are also subject to herding behavior.

- A third natural mechanism of endogenous dynamics is based on market interactions. Let us assume a situation in which technical traders can switch between several financial markets. A market may temporarily become unstable if it attracts numerous chartists from other markets. However, when chartists leave the market again - e.g. when other markets appear to be more profitable a period of convergence sets in. Models along these lines have been proposed by Westerhoff (2004), Chiarella et al. (2005) and Westerhoff and Dieci (2006).

The contribution our paper makes is as follows: we develop a novel financial market model with five different types of agents. Technical 
traders believe in the persistence of bull and bear markets. For instance, these traders optimistically buy assets in a bull market. In contrast, fundamental traders expect prices to return towards fundamental values. In a situation where the market is overvalued (i.e. in a bull market), fundamentalists submit selling orders. Although these two building blocks are standard in the literature, we generalize them in our paper. First, speculators react asymmetrically in bull and bear markets. Here is an example: fundamentalists may trade more (less) aggressively when an asset is overvalued by 10 percent than when it is undervalued by 10 percent $^{2}$. Second, some speculators determine the size of their orders using linear trading rules. However, other speculators simply keep the size of their orders constant (always trading the same amount of assets) and only determine the direction of trade with their pertinent trading philosophy. Hence, there are two types of technical and two types of fundamental traders. Finally, a market maker, the fifth type of agent, adjusts prices with respect to excess demand in the usual way.

Interestingly, our simple setup constitutes a one-dimensional discontinuous dynamical system which is sufficient to generate a very rich set of dynamical phenomena, including, for instance, irregular fluctuations between bull and bear market regimes, as observed in real markets and first modeled by Day and Huang (1990), yet in a different model environment. This does not, however, imply that the established and sophisticated mechanisms mentioned above do not play an important role in explaining the dynamics of financial markets. It does, however, demonstrate that at least part of the dynamics of financial markets may be due to rather simple deterministic mechanisms. In addition, our paper shows the relevance of discontinuous maps to the analysis of financial market dynamics, a rather new field of applied mathematics that has not yet yielded many results. Nevertheless, note that there are already several interesting economic models that feature piecewise-smooth or discontinuous maps, for example, the pioneering works by Day $(1982,1994)$, Day and Shafer (1987), Day and Pianigiani (1991), which have also been used recently in Metcaf (2008), Böhm and Kaas (2000). It is also worth mentioning the works by Hommes $(1991,1995)$ and Hommes and Nusse (1991, 1995). Discontinuous models are furthermore discussed in Puu and Sushko (2002) and Tramontana et al. (2009a,b,c).

The bifurcations occurring in a piecewise-smooth system may be quite different from those occurring in a smooth one. In fact, in the case of a piecewise-linear system (as in our model) the existing bifur-

\footnotetext{
${ }^{2}$ Such a modelling device is also used in Zhu et al. (2009).
} 
cations are either border-collision ${ }^{3}$ or contact bifurcations ${ }^{4}$, as the local bifurcations associated with the eigenvalues are always degenerate. The dynamic effects of such bifurcations may differ depending on the nature of the invariant sets and the global properties of the map. The term border-collision bifurcation was used for the first time by Nusse and Yorke (1992, 1995), and is now widely used in this context (i.e. for piecewise smooth maps). These bifurcations have been studied in recent years, mainly due to their relevant applications in engineering. The one-dimensional piecewise linear case, continuous and discontinuous, was considered by Banerjee et al. (2000), Jain and Banerjee (2003), Avrutin and Schanz (2006, 2008), Avrutin et al. (2006) and Di Bernardo et al. (2008).

However, this simple subject (bifurcations occurring in one-dimensional piecewise-linear discontinuous maps) has still not been studied completely. In this paper we will be faced with some new cases that, to our knowledge, have not yet been considered in the existing literature. This case (called Case IV), which will be described in the last subsection of the paper, deals with the dynamics in the case of negatives slopes in the components and increasing jump of opposite signs (i.e. from a negative to a positive value), for which we can give a complete characterization. In fact, the simple (linear) components in the description of the model allow for a full analytical study on the possible dynamics. Moreover, particular cases that are often neglected in the literature, may sometimes become relevant, and deserve particular attention. Here we have considered and completely described one such case: the case with slopes both equal to +1 in the components (called Case II).

Obviously, these mathematical results go beyond the economic model we propose in this paper, i.e. they are useful in general to characterize the dynamics of discontinuous maps. In this paper we analyze the deterministic skeletons of more elaborate stochastic versions of our approach. Our deterministic model can already explain some stylized facts of financial markets such as bubbles and crashes and excess volatility. However, a better matching of the stylized facts would require the inclusion of some kind of exogenous noise. For instance, one could add dynamic noise to the traders' demand functions or randomize the traders' reaction coefficients (see Westerhoff and Franke 2009 for an example). Of course, such a model calibration would be most welcome since it helps

\footnotetext{
${ }^{3} \mathrm{~A}$ border-collision is classified as any contact between an invariant set of a map and the border of its region of definition.

${ }^{4}$ According to Fournier-Prunaret et al. (1994) and Mira et al. (1996) a contact bifurcation occurs when two invariant sets of a different nature have a contact in one or more points.
} 
us to identify relevant parameter regions. To keep the paper concise, we stick to deterministic models and leave stochastic extensions of our model for future work.

The remainder of our paper is organized as follows. In section 2, we present our model, derive the dynamical system governing the evolution of prices, and single out a few interesting economic scenarios for our model. In section 3, we explore these scenarios classified into four cases. The results are given analytically in a number of theorems, while graphical and numerical tools are used to illustrate the different cases and show a number of simulations. The last section concludes the paper and offers interesting extensions and directions for future work.

\section{A discontinuous model of a financial market}

In this section, we develop a simple financial market model with five types of agent: a market maker who adjusts prices with respect to order imbalances, two types of technical traders who bet on the persistence of bull and bear markets and two types of fundamental traders who believe in mean reversion. There are two types of technical and fundamental traders since some of them determine the size of their orders on the basis of standard (conventional) linear trading rules while others always prefer to trade a fixed amount of assets. Speculators may also react differently to situations where markets are over- or undervalued. Next, we formalize our model, show that the dynamics are due to a simple one-dimensional discontinuous map and define a few economic scenarios for later analysis.

\subsection{Setup}

Let us turn to the details of the model. A market maker mediates transactions out of equilibrium and adjusts prices with respect to excess demand. To be precise, the market maker quotes the log of price $P$ for period $t+1$ as

$$
P_{t+1}=P_{t}+a\left(D_{t}^{C, 1}+D_{t}^{C, 2}+D_{t}^{F, 1}+D_{t}^{F, 2}\right),
$$

where $a$ is a positive price adjustment parameter. The orders placed by the two types of technical trader are indicated by $D_{t}^{C, 1}$ and $D_{t}^{C, 2}$, while the orders placed by the two types of fundamental trader are represented by $D_{t}^{F, 1}$ and $D_{t}^{F, 2}$, respectively. Hence, prices increase if buying exceeds selling, and vice versa. Such a price adjustment rule is used in many models, including Beja and Goldman (1980), Day and Huang (1990) and Farmer and Joshi (2002). Without loss of generality, we set the scaling parameter to $a=1$. 
Following Day and Huang (1990), chartists believe in the persistence of bull and bear markets. The orders placed by the first type of chartists are expressed as

$$
D_{t}^{C, 1}:=\left\{\begin{array}{lll}
c^{1, a}\left(P_{t}-F\right) & \text { if } & P_{t}-F \geq 0 \\
c^{1, b}\left(P_{t}-F\right) & \text { if } & P_{t}-F<0
\end{array},\right.
$$

where $c^{1, a}$ and $c^{1, b}$ are positive reaction parameters and $F$ is the log of the fundamental value. Hence, type 1 chartists submit buying (selling) orders when prices are above (below) the fundamental value. Note that the size of these orders may be asymmetric with respect to a bull or bear market situation. For instance, for $c^{1, a}>c^{1, b}$ this type of traders bets more heavily in a bull market than in a bear market. Moreover, similar formulations for the behavior of chartists have been used, for instance, by Brock and Hommes (1998) and He and Westerhoff (2005). Moreover, Boswijk et al. (2007) estimate Brock and Hommes' model using yearly S\&P 500 data and find significant evidence for such a kind of trading behavior.

Type 2 chartists submit orders according to

$$
D_{t}^{C, 2}:=\left\{\begin{array}{lcc}
c^{2, a} & \text { if } & P_{t}-F>0 \\
0 & \text { if } & P_{t}-F=0 \\
-c^{2, b} & \text { if } & P_{t}-F<0
\end{array} .\right.
$$

The order size in the bull market is given by $c^{2, a}>0$ while in the bear market it is given by $c^{2, b}>0$. Compared to (2), type 2 chartists also expect bull and bear markets to continue. However, the size of their orders does not depend on the deviation from the fundamental value but it is fixed. Also Lux (1998) considers a financial market model with optimistic and pessimistic traders who always seek to buy or sell a fixed amount of assets. Note that (3) implies that in the (special) case where prices are equal to the fundamental value, type 2 chartists do not submit orders.

Fundamentalists believe that prices return to their fundamental value in the long run. The orders placed by type 1 fundamentalists are formalized as

$$
D_{t}^{F, 1}:=\left\{\begin{array}{lll}
f^{1, a}\left(F-P_{t}\right) & \text { if } & F-P_{t}>0 \\
f^{1, b}\left(F-P_{t}\right) & \text { if } & F-P_{t} \leq 0
\end{array},\right.
$$

where $f^{1, a}$ and $f^{1, b}$ are positive reaction parameters. For instance, if prices are above the fundamental value, the market is regarded as overvalued, causing type 1 fundamentalists to sell assets (as in Brock and Hommes 1998 or Lux 1998). Again, also type 1 fundamentalists may 
react asymmetrically to over- and undervalued markets, i.e. $f^{1, a}$ and $f^{1, b}$ may be unequal ${ }^{5}$.

Finally, the orders placed by type 2 fundamentalists amount to

$$
D_{t}^{F, 2}:=\left\{\begin{array}{lcc}
f^{2, a} & \text { if } & F-P_{t}>0 \\
0 & \text { if } & F-P_{t}=0 \\
-f^{2, b} & \text { if } & F-P_{t}<0
\end{array} .\right.
$$

Should the market be undervalued, type 2 fundamentalists submit buying orders amounting to $f^{2, a}>0$. Note that they become inactive in the (special) case in which the price just mirrors its fundamental value. Otherwise, their selling orders are given by $f^{2, b}>0$.

Although formulations (2) to (5) are simple descriptions of the behavior of traders, they may be regarded as natural generalizations of the general content of related models. Here we show that such a still simple trading process is sufficient to generate interesting price dynamics.

\subsection{The dynamical system}

Introducing $\widetilde{P}_{t}=P_{t}-F$, in $(2)$ - (5) we obtain the following dynamical system

$$
\widetilde{P}_{t+1}=\left\{\begin{array}{lcc}
\left(1+c^{1, a}-f^{1, b}\right) \widetilde{P}_{t}+c^{2, a}-f^{2, b} & \text { if } & \widetilde{P}_{t}>0 \\
0 & \text { if } & \widetilde{P}_{t}=0 . \\
\left(1+c^{1, b}-f^{1, a}\right) \widetilde{P}_{t}-c^{2, b}+f^{2, a} & \text { if } & \widetilde{P}_{t}<0
\end{array}\right.
$$

To simplify the notation, let us furthermore define the slopes as

$$
s_{R}=1+c^{1, a}-f^{1, b}, \quad s_{L}=1+c^{1, b}-f^{1, a}
$$

and the intercepts as

$$
m_{R}=c^{2, a}-f^{2, b}, \quad m_{L}=f^{2, a}-c^{2, b} .
$$

We then obtain

$$
\widetilde{P}_{t+1}=T\left(\widetilde{P}_{t}\right)=\left\{\begin{array}{llc}
f_{R}\left(\widetilde{P}_{t}\right)=s_{R} \widetilde{P}_{t}+m_{R} & \text { if } & \widetilde{P}_{t}>0 \\
0 & \text { if } & \widetilde{P}_{t}=0 \\
f_{L}\left(\widetilde{P}_{t}\right)=s_{L} \widetilde{P}_{t}+m_{L} & \text { if } & \widetilde{P}_{t}<0
\end{array}\right.
$$

which is a one-dimensional and, in general, discontinuous map.

\footnotetext{
${ }^{5}$ Note that fundamentalists and chartists always trade in opposite directions (the main difference between (2) and (4) lies in the "if "condition). Our chartists may thus also be regarded as anti-fundamentalists.
} 
Note that in our formulation of the map, except for what is called "Case I" below, we have that $\widetilde{P}_{t}=0$ is a very particular fixed point: the map is not smooth in it, and the dynamics of points close to it are associated with the values of the jump in this point, which is also a discontinuity point. That is, points on its right side behave as the point $f_{R}(0)=m_{R}$, while points on its left side behave as $f_{L}(0)=m_{L}$. Of such a particular fixed point we may look at its stable set $W^{S}(0)$ (which is necessarily a numerable set), and we shall see how its structure changes in the different cases here analyzed. This point is also important as a discontinuity point in the global analysis, because it behaves as a critical point for smooth maps: as we shall see, both the values $m_{R}$ and $m_{L}$ will be involved in the bifurcations (they assume the same role of the critical values in the smooth cases).

Also it is worth noting that, of the four parameters we have in (9), one may be set to 1 by rescaling the independent variable. Thus only three parameters are really important to classify the dynamics and bifurcations (for example, $s_{R}, s_{L}$ and the ratio $m_{R} / m_{L}$ ). However, we prefer to keep all four parameters as they are given, to have a suitable interpretation in the applied context (and the reduction to three parameters in the formulas can always be done a posteriori, if needed).

\section{$2.3 \quad$ Economic scenarios}

Note that all eight reaction parameters of (2)-(5) are positive. Hence, the four model parameters given in (7) and (8) can take any values. A full analytical treatment of model (9) is beyond the scope of the current paper. Instead, we next single out four economic scenarios which we will then investigate in detail in section 3 . The first two scenarios are rather special cases whereas the other two scenarios are more general.

We obtain a first (special) case, called Case I, if we omit type 2 traders. Setting $m_{R}=c^{2, a}-f^{2, b}=0$ and $m_{L}=f^{2, a}-c^{2, b}=0$, the model turns into the continuous map

$$
\text { (Case I) } \widetilde{P}_{t+1}=\left\{\begin{array}{lll}
f_{R}\left(\widetilde{P}_{t}\right)=s_{R} \widetilde{P}_{t} & \text { if } & \widetilde{P}_{t} \geq 0 \\
f_{L}\left(\widetilde{P}_{t}\right)=s_{L} \widetilde{P}_{t} & \text { if } & \widetilde{P}_{t} \leq 0
\end{array} .\right.
$$

While the dynamic properties of (10) are trivial, a brief systematic summary of this case may be helpful in understanding the other cases.

Excluding type 1 traders, i.e. fixing $s_{R}=1+c^{1, a}-f^{1, b}=1$ and $s_{L}=1+c^{1, b}-f^{1, a}=1$, the model in (9) is simplified to

$$
\text { (Case II) } \widetilde{P}_{t+1}=\left\{\begin{array}{lll}
f_{R}\left(\widetilde{P}_{t}\right)=\widetilde{P}_{t}+m_{R} & \text { if } & \widetilde{P}_{t}>0 \\
0 & \text { if } & \widetilde{P}_{t}=0 \\
f_{L}\left(\widetilde{P}_{t}\right)=\widetilde{P}_{t}+m_{L} & \text { if } & \widetilde{P}_{t}<0
\end{array}\right.
$$


We call this special situation Case II. As we shall see in the next section, the dynamics are only relevant if $m_{L}>0$ and $m_{R}<0$, i.e. type 2 fundamentalists are more aggressive than type 2 chartists. Obviously, the opposite case, that is a dominance of type 2 chartists over type 2 fundamentalists, leads directly to an explosion of the model dynamics.

Two further interesting special cases, yet not analyzed in this paper, include models where there are either only chartists or only fundamentalists. It is immediately seen that the dynamics always explodes if there are only type 1 and type 2 chartists, which is also consistent with basic economic intuition ${ }^{6}$. However, the case in which there are only type 1 and type 2 fundamentalists is more complicated ${ }^{7}$. At least for some parameter constellation a model with two different types of fundamentalists can generate complex endogenous dynamics. Note that pairing destabilizing chartists with destabilizing fundamentalists does not necessarily lead to unbounded dynamics. In this sense, also "destabilizing" chartist can contribute to financial market stability.

Case III is more general and assumes existence of all possible trader types. For map (9) we consider restrictions $s_{R}>1, s_{L}>1, m_{R}<0$ and $m_{L}>0$, which corresponds to a situation in which type 1 chartists dominate type 1 fundamentalists but type 2 chartists are dominated by type 2 fundamentalists. Dynamically, only chaotic behavior can occur (a similar situation was considered in the paper by Tramontana et al. $(2009 \mathrm{c}))$.

Finally, in Case IV we consider the parameter space that is generic in the negative slopes: $s_{R}<0, s_{L}<0$ and increasing jump, from $m_{L}<0$ to $m_{R}>0$. In economic terms, this means that type 1 fundamentalists trade (much) more forcefully than type 1 chartists. The term "(much) more forcefully" reflects the fact that $f^{1, b}-c^{1, a}>1$ and $f^{1, a}-c^{1, b}>1$. In addition, type 2 fundamentalists are less aggressive than type 2 chartists. The study of such a dynamic system, which is new, is fully described in our paper. We shall see a rich variety of possible behaviors: bistability of equilibria, or convergence to equilibria coexisting with explosive trajectories, or bounded and chaotic dynamics.

\footnotetext{
${ }^{6}$ This case requires that $f^{1, a}=f^{1, b}=0$. The slopes of the map are both positive: $s_{R}=1+c^{1, a}>0$ and $s_{L}=1+c^{1, b}>0$. The offsets are such that: $m_{R}=c^{2, a}>0$ and $m_{L}=-c^{2, b}<0$.

${ }^{7}$ This case requires that $c^{1, a}=c^{1, b}=0$. The slopes of the map are: $s_{R}=$ $1-f^{1, b}<1$ and $s_{L}=1-f^{1, a}<1$. The offsets are one negative and one positive: $m_{R}=-f^{2, b}<0$ and $m_{L}=f^{2, a}>0$.
} 


\section{The dynamics of the model}

In this section, we explore the four economic scenarios singled out in the previous section. Due to the simple structure of our model, several results are analytical and, as already stated in the previous sections, the analyses of Cases II and IV are the most innovative ones. The use of graphical and numerical tools will help us to present the results and simulate possible trajectories of the applied model.

\subsection{Case I}

In this section we describe the dynamics in the particular case in which

$$
m_{L}=m_{R}=0
$$

so that the model reduces to the continuous map given in (10), where the two slopes $s_{R}$ and $s_{L}$ can have any sign. Obviously, the only fixed point of the map is point $\widetilde{P}_{t}=0$, and the trajectories either converge to this point or diverge. The complete description of all possible cases is summarized in the following figure, where, depending on the sign and modulus of the slopes, we have different dynamic behaviors.

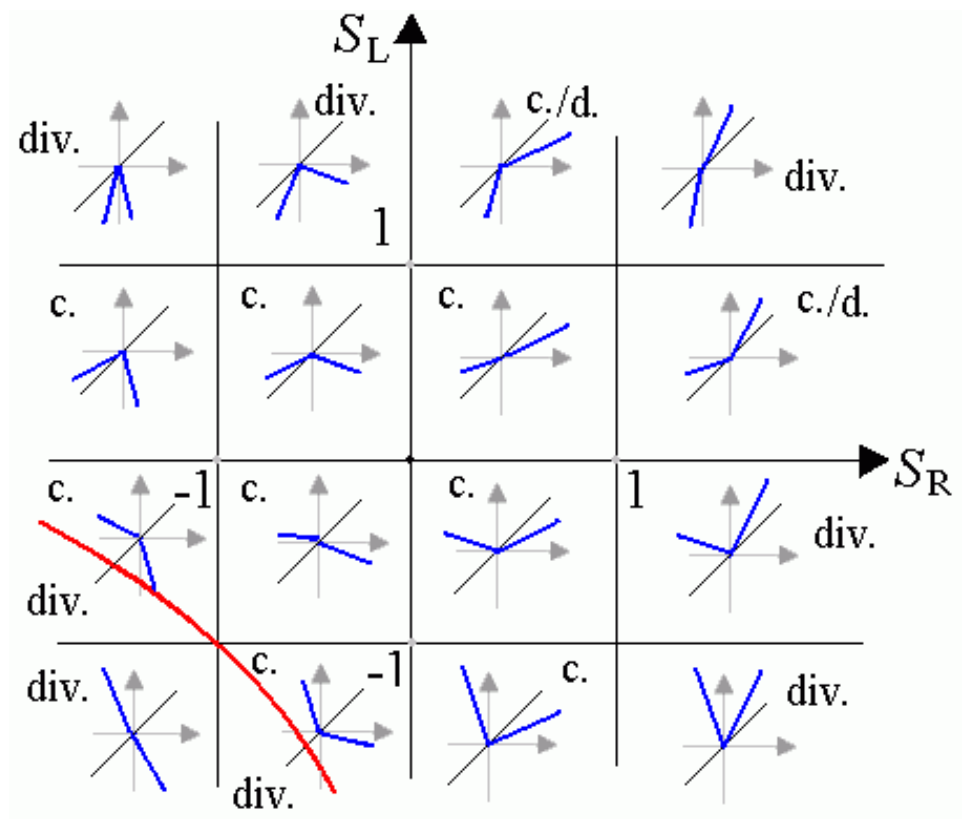

Fig. 1 Qualitative shape of the map for $m_{R}=m_{L}=0$ depending on parameters $\left(s_{R}, s_{L}\right)$. "c." stands for convergence while "div." indicates divergence.

It is clear that when both slopes are in modulus less than one, the origin is an attracting fixed point (the regions marked by "c." in Fig. 1), while different dynamics may occur in other parts of the parameter 
space $\left(s_{R}, s_{L}\right)$. From Fig. 1 we can see that divergent dynamics can occur in some cases only (the regions marked by "div." in Fig. 1), while in two regions, characterized by $\left(s_{R}>1,0<s_{L}<1\right)$ and by $\left(0<s_{R}<1, s_{L}>1\right)$, we have convergence on one side and divergence on the other. The arc of the curve in the lower-left part of the graph is the curve of equation $s_{R} s_{L}=1$. In the two regions crossed by this curve the dynamic behavior (convergence or divergence) depends on the product of the slopes. The results are summarized in the following Theorem, proof of which is given in the Appendix.

Theorem 1. Let $m_{R}=m_{L}=0$ in map (9). Then:

- if $\left|s_{R, L}\right|<1$, or $0<s_{R}<1$ and $s_{L}<-1$, or $0<s_{L}<1$ and $s_{R}<-1$, all trajectories are convergent to $\widetilde{P}=0$;

- if $\left|s_{R, L}\right|>1$, or $s_{R}>1$ and $-1<s_{L}<0$, or $s_{L}>1$ and $-1<s_{R}<0$, all trajectories are divergent;

- if $s_{R}>1$ and $0<s_{L}<1$, any $\widetilde{P}>0$ has a divergent trajectory while any $\widetilde{P}<0$ is convergent to $\widetilde{P}=0$;

- if $s_{L} \geq 1$ and $-1<s_{R}<0$, any $\widetilde{P}<0$ has a divergent trajectory while any $\widetilde{P}>0$ is convergent to $\widetilde{P}=0$;

- if $s_{R, L}<0$, for $s_{R} s_{L}<1$ all trajectories are convergent to $\widetilde{P}=0$, while for $s_{R} s_{L}>1$ all trajectories are divergent;

- if $s_{L}=1$ (resp. $s_{R}=1$ ) all points $\widetilde{P}<0$ (resp. $\widetilde{P}>0$ ) are fixed;

- if $s_{L}=-1$ and $\left|s_{R}\right|<1$ (resp. $\left|s_{R}\right|>1$ ) all points are convergent to $\widetilde{P}=0$ (resp. divergent);

- if $s_{R}=-1$ and $\left|s_{L}\right|<1$ (resp. $\left|s_{L}\right|>1$ ) all points are convergent to $\widetilde{P}=0$ (resp. divergent);

- if $s_{L}=s_{R}=-1$ all points are periodic of period 2;

- if $s_{L}=0$ (resp. $\left.s_{R}=0\right)$ all points $\widetilde{P}<0$ (resp. $\left.\widetilde{P}>0\right)$ are mapped into the fixed point $\widetilde{P}=0$.

Of course, neither a globally repelling nor attracting fixed point is a compelling description of the dynamics of financial markets. However, this brief investigation helps us to understand the more complicated cases discussed next. However, we add that Westerhoff and Franke (2009) proposed a similar model containing only type 1 chartists and type 1 fundamentalists that is able to match some important stylized facts of financial markets surprisingly well. Due to intrinsic multiplicative noise in their model, the dynamics fluctuates stochastically between periods of monotonic convergence and monotonic divergence, and thus price time series resemble random walks and returns display no significant autocorrelation. In other words, even simple models such as our map (10) may be useful in understanding the dynamics of financial markets. 


\subsection{Case II}

In this section we describe the dynamics in the particular case in which

$$
s_{L}=s_{R}=+1
$$

so that the model reduces to the discontinuous map given in (11).
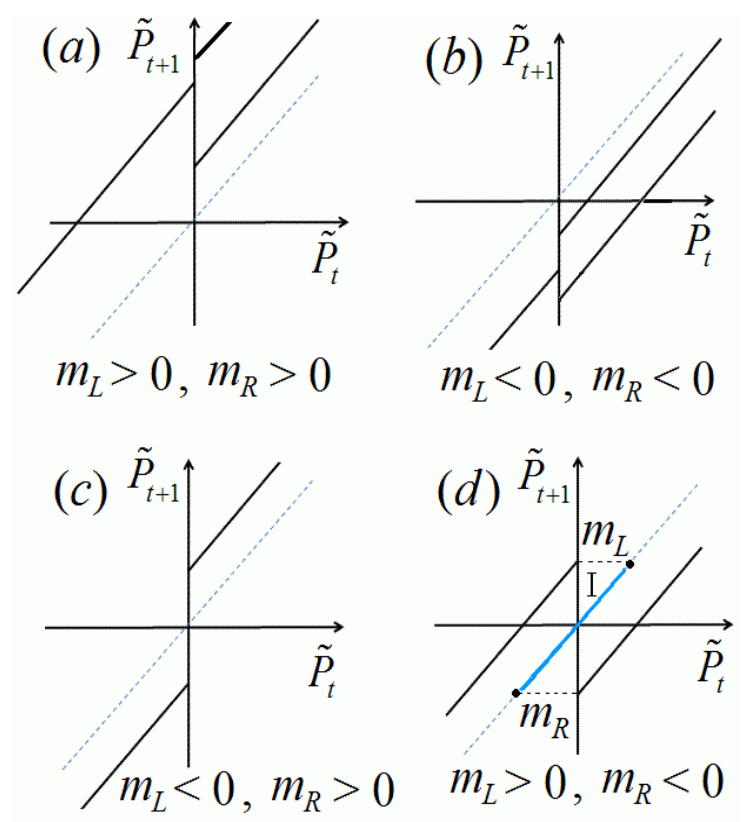

Fig. 2 Qualitative shape of the map for $s_{L}=s_{R}=1$. In cases (a) and (b) two lines are drawn on the right to show that it is irrelevant whether

$$
m_{L}>m_{R} \text { or } m_{L}<m_{R} \text {. }
$$

The shape of the map, depending on all possible cases of the sign of $m_{L}$ and $m_{R}$, is illustrated in Fig. 2. It can immediately be seen from the figure that the trajectories are divergent, with the exception of the situation shown in Fig. 2(d). Thus we are led, in this section, to describe the dynamics in this particular case assuming $m_{L}>0$ and $m_{R}<0$. One preliminary remark is that no fixed point $\widetilde{P} \neq 0$ exists, and $I=\left[m_{R}, m_{L}\right]$ is an invariant absorbing interval (here absorbing means that any initial condition, i.c. henceforth, outside $I$ has a trajectory which is mapped in $I$ in a finite number of iterations).

Note that the stable set $W^{S}(0)$ of $\widetilde{P}=0$ consists of only this point in the case of Fig. 2(c), while it includes a numerable set of points in the other cases: we have $W^{S}(0)=\left\{-k m_{L}, k \geq 0\right\}$ in the case of Fig. 2(a), $W^{S}(0)=\left\{-k m_{R}, k \geq 0\right\}$ in the case of Fig. 2(b), and $W^{S}(0)=\left\{-k m_{L},-k m_{R}, k \geq 1\right.$, and related preimages $\}$ in the case of Fig. 2(d). 
Let us explore the dynamics for $m_{L}>0$ and $m_{R}<0$. As the slopes of the two components are both equal to +1 we cannot have states which are "attracting", but neither repelling nor divergent. In fact, all of the points in $I$ not belonging to $W^{S}(0)$ have a similar fate: either they are all periodic of the same period or they all have quasiperiodic trajectories, which are limited inside the interval forever, albeit without a chaotic behavior. The reason for the non-existence of chaos is that there is no sensitivity with respect to the initial conditions: points starting close together will have trajectories similarly close to each other for any iteration. The distinguishing role for periodic or quasiperiodic trajectories in $I$ is simply the ratio between the two values: $m_{L} / m_{R}$ being rational or irrational, respectively. This result, and the exact computation of the period, is stated in the following Theorem, the proof of which is given in the Appendix.

Theorem 2. Let $s_{R}=s_{L}=1$ in map (9). Then the trajectory of any $\widetilde{P} \notin W^{S}(0)$ is divergent, with the exception of the case $m_{R}<0$ and $m_{L}>0$ at which $I=\left[m_{R}, m_{L}\right]$ is an invariant absorbing interval. All points in $I \backslash W^{S}(0)$ are either periodic or quasiperiodic with trajectories dense in I. All points outside I have a trajectory entering I in a finite number of iterations. Define $\rho=\frac{m_{L}}{-m_{R}}$. Then:

- if $\rho$ is rational let $\frac{m_{L}}{-m_{R}}=\frac{n}{k}$, where $n$ and $k$ are integers with no common divisors, all the orbits in $I \backslash W^{S}(0)$ have period $(n+k)$, where $k$ is the number of periodic points on the $L$ side and $n$ is the number of periodic points on the $R$ side;

- if $\rho$ is irrational, all orbits in $I \backslash W^{S}(0)$ are quasiperiodic.

Thus considering the non-divergent region in the parameter plane $\left(m_{R}, m_{L}\right)$ we find that it is filled with straight lines $m_{L}=-\rho m_{R}$ (see Fig. 3). Also, for each line there is a corresponding (in the phase space) absorbing interval $I$ filled with periodic orbits when $\rho$ is rational, or quasiperiodic orbits when $\rho$ is irrational.

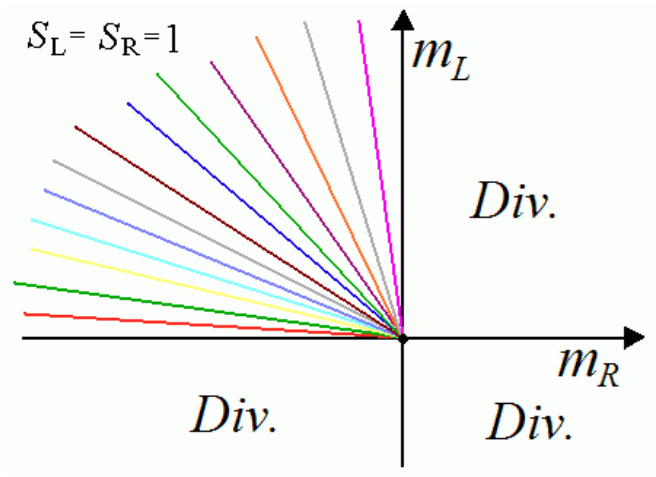

Fig. 3 Qualitative behavior of the map in phase space $\left(m_{R}, m_{L}\right)$ for $s_{R}=s_{L}=1$. If slope $\rho$ of a line is rational (resp. irrational), the dynamics 
are periodic (resp. quasiperiodic).

A number of examples are given in Fig. 4 (always for the case $m_{L}>0$ and $\left.m_{R}<0\right)$. Fig. 4(a) is obtained using $F=0 ; a=1 ; c^{1, a}=c^{1, b}=0$; $c^{2, a}=c^{2, b}=0.2 ; f^{1, a}=f^{1, b}=0$ and $f^{2, a}=f^{2, b}=0.3$. We have $m_{L}=0.1$ and $m_{R}=-0.1$, so $\frac{m_{L}}{-m_{R}}=\frac{1}{1}$, and we have a $2-$ cycle with one point on the left and one point on the right. If we increase the value of $f^{2, a}$ to $0.4, m_{L}$ becomes equal to 0.2 and $\frac{m_{L}}{-m_{R}}=\frac{2}{1}$. This is the case shown in Fig. 4(b) where we have a 3-cycle with one point on the left and two points on the right. Fig. 4(c) shows the case with $f^{2, a}=0.41$, which implies that $\frac{m_{L}}{-m_{R}}=\frac{21}{10}$; the cycle is characterized by period 31 with 10 periodic points located on the left and 21 on the right. Note that Fig. 4(c) depicts very interesting price dynamics. The repeated and consistent up trend price swings are due to the interactions between the fundamentalists and chartists, and in particular due to the "overreaction" of fundamentalists ${ }^{8}$. Finally, Fig. 4(d) shows a case in which $\rho$ is irrational. In this case, $f^{2, a}=\sqrt{2} / 3$ and the orbits are quasiperiodic.

\footnotetext{
${ }^{8}$ However, we observe qualitatively similar price dynamics for, e.g., $f^{2, a}=0.3$ and $f^{2, b}=0.29$, i.e. we can also hold $f^{2, a}$ constant and decrease $f^{2, b}$. We thank an anonymous referee for pointing this out to us.
} 


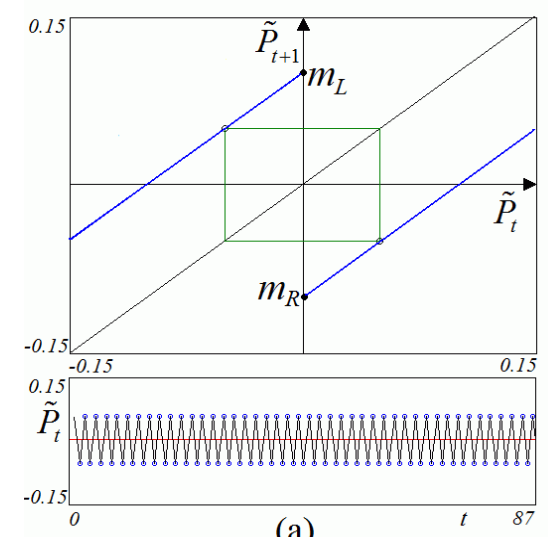

(a)

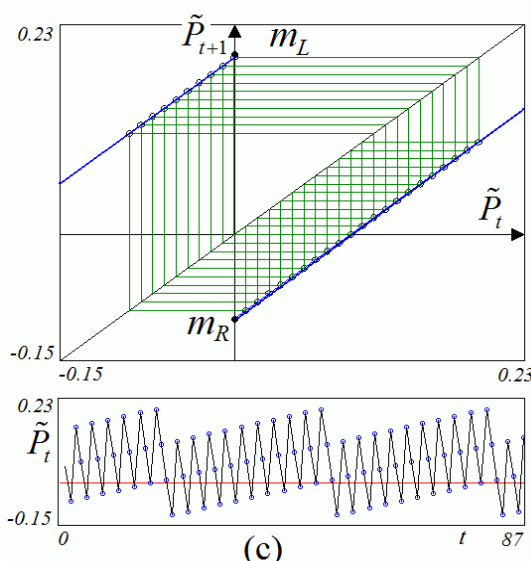

(c)

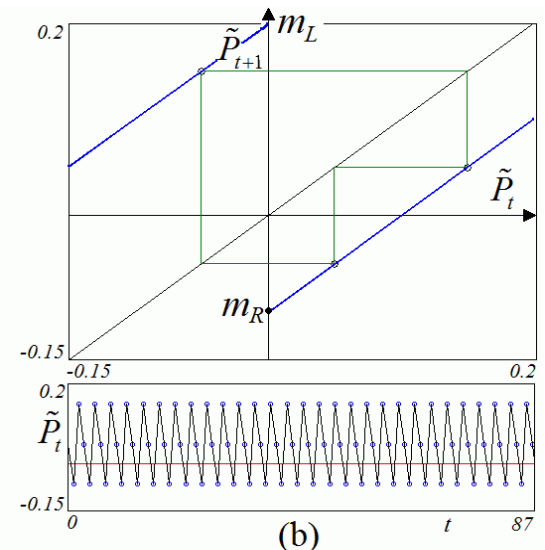

(b)
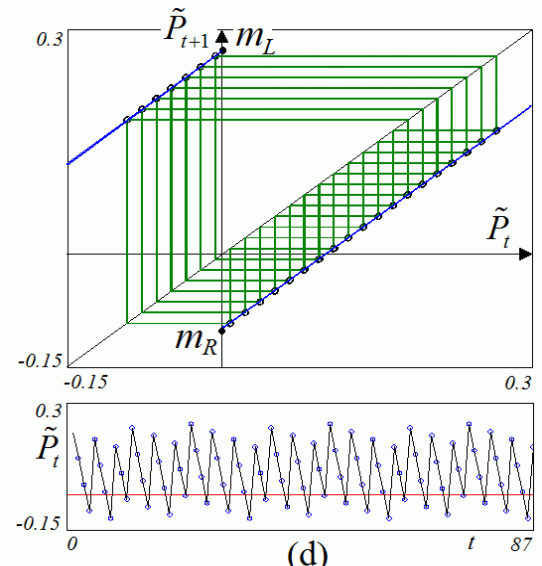

(d)

Fig. 4 Examples of periodic and quasi-periodic trajectories. A cycle of period 2 in (a), of period 3 in (b) and of period 31 in (c). An aperiodic trajectory is shown in (d). Parameter $f^{2, a}$ is increased from 0.3 to 0.4 to 0.41 and to $\sqrt{2} / 3$. The remaining parameters are constant and given in

Section 3.2.

From an economic point of view, it is clearly obvious here that even an extremely simple financial market model such as ours is able to generate endogenous asset price dynamics. In the current setting, chartists and fundamentalists always seek to trade constant amounts of assets, a regime which is incompatible with fixed point dynamics. This observation may further help us to understand why financial markets are so (excessively) volatile.

\subsection{Case III}

Let us consider the general case (9), where both slopes $s_{R}$ and $s_{L}$ are positive and larger than 1 , that is: $c^{1, a}-f^{1, b}>0$ and $c^{1, b}-f^{1, a}>0$, while $m_{R}=c^{2, a}-f^{2, b}<0$ and $m_{L}=f^{2, a}-c^{2, b}>0$ (the qualitative shape of the map is shown in Fig. 5).

In this case, no stable cycle can exist because, given a cycle of period 
$k$ with $l$ points in the $L$ side and the remaining $k-l$ points on the $R$ side, the eigenvalue (or slope) of the $k$-cycle is given by $S=s_{L}^{l} s_{R}^{k-l}>1$. This proves that when bounded dynamics are possible, the trajectories can only be chaotic. In fact, contrary to what occurs in Case II, here the slopes are such that the existing cycles are all repelling and the Lyapunov exponent, computed in aperiodic trajectories, is necessarily positive. Also we recall that in piecewise linear maps chaotic attractors are always of so-called robust chaos, as we shall see below.

The restrictions considered in this case also imply that two (locally unstable) fixed points with $\widetilde{P} \neq 0$ always exist: a negative valued fixed point and a positive one, given by

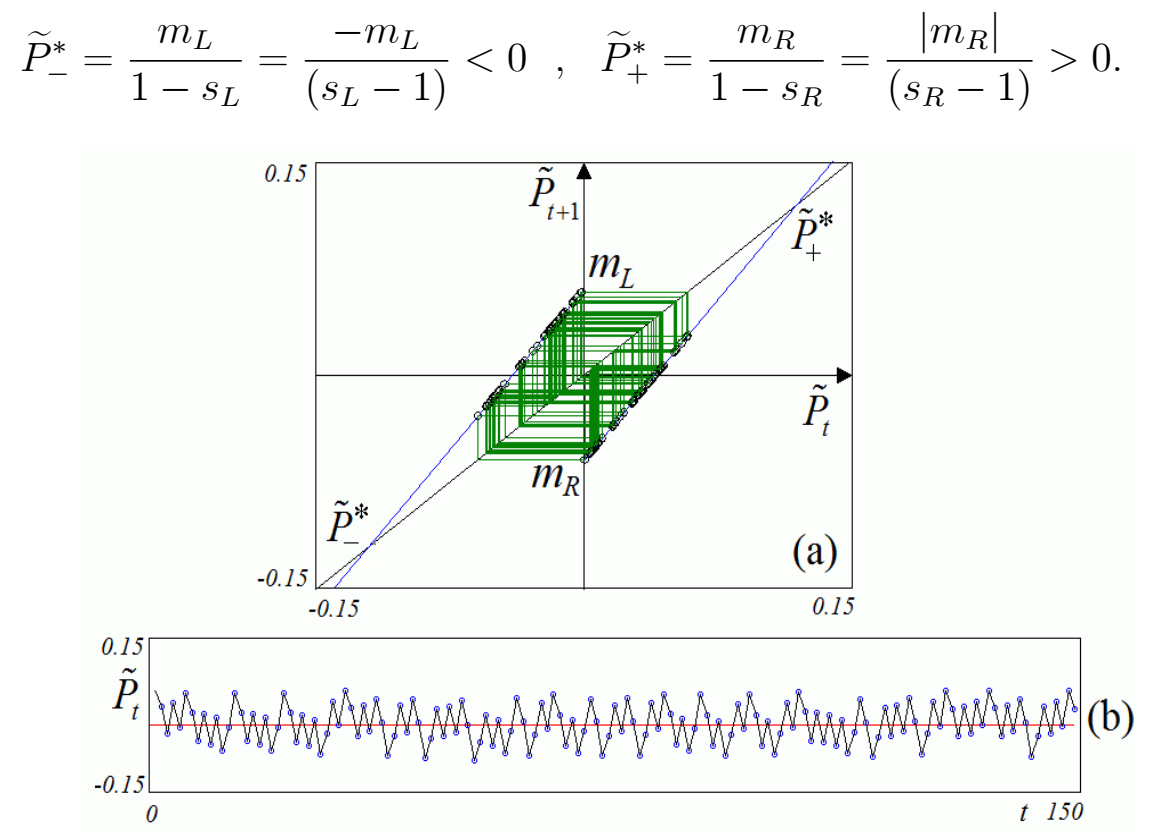

Fig. 5 Set of parameters: $F=0 ; a=1 ; c^{1, a}=c^{1, b}=0.5 ; c^{2, a}=c^{2, b}=0.2$;

$$
f^{1, a}=f^{1, b}=0 \text { and } f^{2, a}=f^{2, b}=0.3 .
$$

The existence of such unstable fixed points implies that any i.c. (remember that i.c. stands for initial condition) lower than $\widetilde{P}_{-}^{*}$ has a trajectory which is divergent towards $-\infty$, while any i.c. higher than $\widetilde{P}_{+}^{*}$ has a trajectory which is divergent towards $+\infty$. It follows that bounded dynamics can only occur inside interval $] \widetilde{P}_{-}^{*}, \widetilde{P}_{+}^{*}[$, and a necessary and sufficient condition to have bounded trajectories is the following:

$$
\widetilde{P}_{-}^{*}<m_{R} \quad \text { and } \quad \widetilde{P}_{+}^{*}>m_{L} .
$$

Condition (13), which may be rewritten as a function of the parameters as follows:

$$
\frac{m_{L}}{1-s_{L}}<m_{R}<m_{L}\left(1-s_{R}\right)
$$


or:

$$
m_{R}\left(1-s_{L}\right)<m_{L}<\frac{m_{R}}{1-s_{R}},
$$

also states that the interval $I=\left[m_{R}, m_{L}\right]$ is invariant (any point in $I$ has a trajectory which stays in $I$ forever) and absorbing: it attracts the trajectory of all i.c. that are above $\widetilde{P}_{-}^{*}$ and below $\widetilde{P}_{+}^{*}$. In fact, such i.c. have a trajectory which enters interval $I$ in a finite number of steps (the number of steps depends on the two slopes and on width of $I$ ). Thus the basin of attraction of this absorbing interval is given by $B(I)=] \widetilde{P}_{-}^{*}, \widetilde{P}_{+}^{*}[$. In the example shown in Fig. 5(a), the sequence of prices can switch between bull and bear regions without a fixed number of times for which it remains in a particular region (see the time series plot in Fig. 5(b)).

The dynamics of prices inside $I$ is chaotic. Infinitely many cycles exist because equations $T^{k}(\widetilde{P})=\widetilde{P}$ have different solutions for any integer $k$, but all $k$-cycles are unstable (as remarked above) and the attracting set of the map inside the invariant interval $I$ is given by some $n$-cyclical chaotic intervals (with $n \geq 1$ ), bounded by the images of the discontinuity point. What may change in this regime is the number of chaotic components, via homoclinic bifurcations of repelling cycles outside the chaotic intervals, occurring due to the merging of the images of the discontinuity point with such unstable cycles. A detailed description of the bifurcations involving chaotic intervals can be found in Avrutin et al. (2006, 2008a,b, 2009). When the slopes are large enough, the dynamics become chaotic in the whole interval $I=\left[m_{R}, m_{L}\right]$ (i.e. $n=1$, and we have one chaotic interval). In general, when $n$-cyclical chaotic intervals exist, the dynamics of map $T^{n}$ in one invariant segment is topologically conjugated with that of the shift map (that is, equivalently, with that of the tent map), and it is purely chaotic: the periodic points are dense in the $n$-cyclical chaotic intervals, as well as the aperiodic trajectories, and an absolutely continuous invariant measure exists. This is a classical case of pure chaos in intervals of the phase space, and also occurring in an interval of values for each parameter. This is an important property in the applied context, which is often missed. It is well known that this "pure chaos" also exists in smooth models (we may think of several cases modelled, for example, with the logistic map, and cyclical chaotic intervals), but often it cannot occur for an interval of parameter values. Differently, in piecewise-linear models with a chaotic attractor, it is persistent as a function of the parameters, at least in some interval for each parameter, and this property is often called robust chaos. An example is shown in the one-dimensional bifurcation diagram of Fig. 6 (the parameters are the same as in Fig. 5, while $f^{2, b}$ varies between 0.2 and 0.42 ). We remark that $f^{2, b}$ is a component of $m_{L}$, which decreases 
up to the final bifurcation causing the divergence.

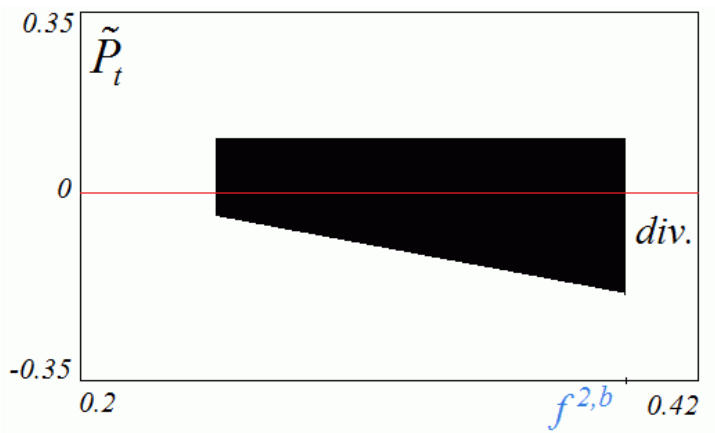

Fig. 6 Bifurcation diagram obtained with the following set of parameters: $F=0 ; a=1 ; c^{1, a}=c^{1, b}=0.5 ; c^{2, a}=c^{2, b}=0.2 ; f^{1, a}=f^{1, b}=0$ and $f^{2, a}=0.3 . f^{2, b}$ varies between 0.2 and 0.42 .

The final bifurcation occurring when $\widetilde{P}_{-}^{*}=m_{R}$ or $\widetilde{P}_{+}^{*}=m_{L}$ is a contact bifurcation, leading to the disappearance of the attractor. The contact occurs due to the merging of one extremum of the invariant absorbing interval $I=\left[m_{R}, m_{L}\right]$ with one unstable fixed point, which is on the boundary of its basin of attraction $B(I)=] \widetilde{P}_{-}^{*}, \widetilde{P}_{+}^{*}[$. This also corresponds to the homoclinic bifurcation of the unstable fixed point involved in the contact. After such a bifurcation, all trajectories are divergent, with the exception of the unstable cycles which persist (unstable) in $I$ (because equations $T^{k}(\widetilde{P})=\widetilde{P}$ have different solutions for any integer $k)$, that is the points belonging to their stable sets and their limit points. This set of points which persists bounded in $I$ is an invariant Cantor set $\Lambda$, and the restriction of map $T$ to $\Lambda$ is chaotic, as rigorously proved in the Appendix. Hence we have proved the following

Theorem 3. Let $s_{R}>1, s_{L}>1, m_{R}<0, m_{L}>0$ in map (9). Then

i) for $\frac{m_{L}}{1-s_{L}}<m_{R}<m_{L}\left(1-s_{R}\right)$ chaotic dynamics occur in chaotic intervals belonging to the invariant absorbing interval $I=\left[m_{R}, m_{L}\right]$ with basin $B(I)=] \widetilde{P}_{-}^{*}, \widetilde{P}_{+}^{*}[$;

ii) for $\frac{m_{L}}{1-s_{L}}=m_{R}$ or $m_{R}=m_{L}\left(1-s_{R}\right)$ fixed point $\widetilde{P}_{-}^{*}$ or $\widetilde{P}_{+}^{*}$ respectively (involved in the contact) becomes homoclinic;

iii) for $\frac{m_{L}}{1-s_{L}}>m_{R}$ or $m_{R}>m_{L}\left(1-s_{R}\right)$ almost all trajectories are divergent, i.e. except for the points of a chaotic repellor $\Lambda$ (a Cantor set of points of zero Lebesque measure in I).

We remark that the number of steps spent by a chaotic trajectory in the bull region or bear region before switching to the other regime is unpredictable, although rough estimates may be associated with the width of the chaotic interval and, especially, to the width of the positive 
parameter $m_{L}$ or that of the negative one $m_{R}$. For example, when the parameters are such that $m_{L}$ (resp. $m_{R}$ ) is quite close to 0 , while the other is further from 0 , the chaotic states spend more iterations in the $L$ (resp. $R$ ) region.
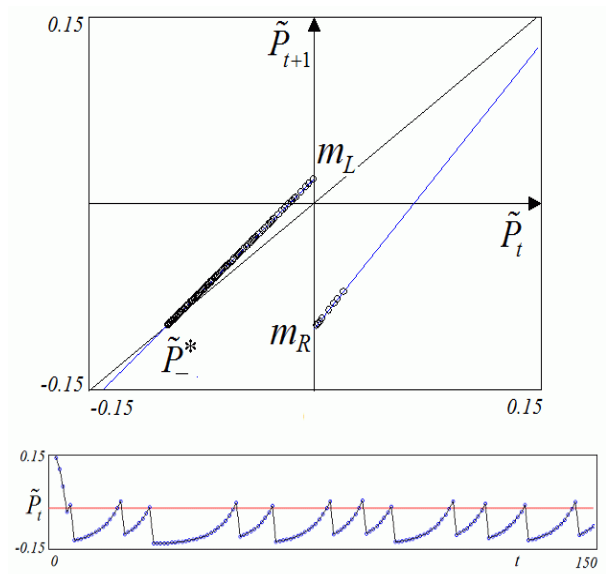

(a)
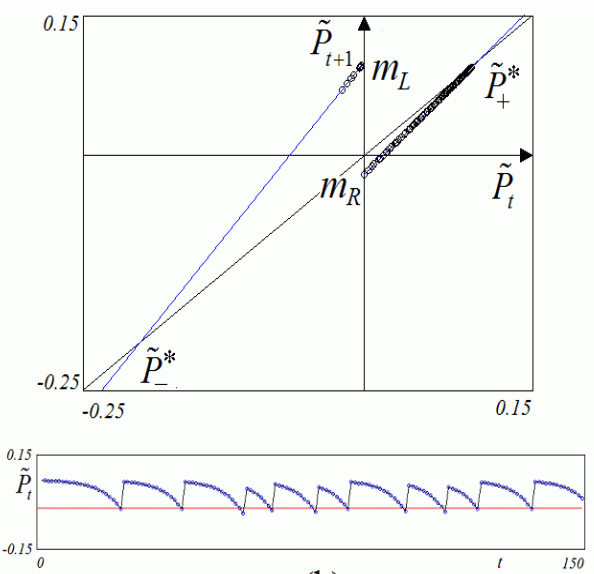

(b)

Fig. 7 Set of parameters: a) $F=0 ; a=1 ; c^{1, a}=0.5 ; c^{1, b}=0.202$; $c^{2, a}=c^{2, b}=0.2 ; f^{1, a}=f^{1, b}=0 ; f^{2, a}=0.22$ and $f^{2, b}=0.3$. b) $F=0$; $a=1 ; c^{1, a}=0.196 ; c^{1, b}=0.5 ; c^{2, a}=0.28 ; c^{2, b}=0.2 ; f^{1, a}=f^{1, b}=0$ and $f^{2, a}=f^{2, b}=0.3$.

Two examples are shown in Fig. 7. Fig. 7(a) is obtained starting from the set of parameters of Fig. 5. Parameter $f^{2, a}$ is then decreased to yield a lower value of $m_{L}$ and $c^{1, b}$ is decreased to change the slope of the left line and have bounded trajectories, using $c^{1, b}=0.202$ and $f^{2, a}=0.22$; while in Fig. 7(b) (always starting from the set of parameters of Fig. $5)$ we have increased $c^{2, a}\left(c^{2, a}=0.28\right)$ and decreased $c^{1, a}\left(c^{1, a}=0.196\right)$ yielding bounded trajectories with $m_{R}$ close to 0 .

We also notice that in this Case III, the stable set $W^{S}(0)$ always include infinitely many preimages which are accumulating on the two other unstable fixed points (given by the points $-m_{L} / s_{L}$ and $-m_{R} / s_{R}$ which are the rank-1 preimages, and their further preimages of any rank), and when the discontinuity point belongs to the invariant chaotic intervals, then also infinitely many preimages of $\widetilde{P}=0$ are dense in the chaotic set. This means that however close to any point of the invariant chaotic set we can find points which ultimately will end up into $\widetilde{P}=0$, but maybe with a long and chaotic transient.

In contrast to Case II, in which periodic or quasiperiodic motion may emerge due to speculators who always seek to trade fixed amounts of assets, Case III with all four types of speculator is able to produce irregular dynamics, even though the traders' transactions still follow 
quite simple rules. However, note that once a market has entered a bull (bear) area, prices always decrease (increase) until they revert to a bear (bull) area. As we will see in the next section, the dynamics of our model may become more complicated in the sense that prices may move erratically upwards or downwards within a bull or a bear market.

\subsection{Case IV}

We now analyze the general case (9) where both slopes $s_{R}$ and $s_{L}$ are negative, that is: $f^{1, b}-c^{1, a}>1$ and $f^{1, a}-c^{1, b}>1$, while $m_{R}=$ $\left(c^{2, a}-f^{2, b}\right)>0$ and $m_{L}=\left(f^{2, a}-c^{2, b}\right)<0$ (the qualitative shape of the map is shown in Fig. 8(a)).

As in the previous case, also the present one is characterized by the existence of two fixed points $\widetilde{P} \neq 0$ as in (13): one on the left $\left(\widetilde{P}_{-}^{*}=\frac{m_{L}}{1-s_{L}}<0\right)$ and one on the right $\left(\widetilde{P}_{+}^{*}=\frac{m_{R}}{1-s_{R}}>0\right)$. Depending on the local stability of these fixed points (which clearly influences global behaviors), we can subdivide Case IV into three subcases: IV-A when both are stable $\left(-1<s_{R, L}<0\right)$; IV-B when one is stable and the other one unstable $\left(s_{R}<-1\right.$ and $-1<s_{L}<0$ or $s_{L}<-1$ and $\left.-1<s_{R}<0\right)$; IV-C when both are unstable $\left(s_{R, L}<-1\right)$. Let us first point out a few properties to be used later.

Property 1. Any point $\widetilde{P}$ in the interval $\left[m_{L}, m_{R}\right]$ has two distinct rank-1 preimages, one on the $R$ side: $f_{R}^{-1}(\widetilde{P})$, and one on the $L$ side: $f_{L}^{-1}(\widetilde{P})$, given by

$$
f_{R}^{-1}(\widetilde{P})=\frac{\widetilde{P}-m_{R}}{s_{R}}>0 \quad, \quad f_{L}^{-1}(\widetilde{P})=\frac{\widetilde{P}-m_{L}}{s_{L}}<0,
$$

while any point $\widetilde{P}>m_{R}$ has only one rank-1 preimage: $f_{L}^{-1}(\widetilde{P})<0$ and, similarly, any point $\widetilde{P}<m_{L}$ has only one rank-1 preimage: $f_{R}^{-1}(\widetilde{P})>0$.

Several properties of map $T$ in this subcase can be deduced from the properties of the second iterate $T^{2}$. It is a map with three discontinuity points, one in the origin and the other two in the rank-1 preimages of the origin: $f_{L}^{-1}(0)=-\frac{m_{L}}{s_{L}}<0$ and $f_{R}^{-1}(0)=-\frac{m_{R}}{s_{R}}>0$ (the qualitative shape is shown in Fig. 8(b)). $T^{2}$ consists of four branches, two of them have slopes $s_{R}^{2}$ and $s_{L}^{2}$, while the two external branches have the same slope $s_{L} s_{R}$. The jump in the origin has extrema $f_{L}\left(m_{L}\right)=m_{L}\left(1+s_{L}\right)$ and $f_{R}\left(m_{R}\right)=m_{R}\left(1+s_{R}\right)$; the jump in the other two discontinuity points is the same, with extrema $m_{R}>0$ from the left and $m_{L}<0$ from the right. As for $s_{L} s_{R} \leq 1$, the second iterate of map $T^{2}$ cannot have intersections with the diagonal different to the two existing fixed points. Then we immediately have the following:

Property 2. A single 2-cycle of $T$ with periodic points $\bar{x}_{1}<0$ and 
$\bar{x}_{2}>0$ can exist (unstable) iff $s_{L} s_{R}>1$ and the cycle satisfying $f_{L}\left(\bar{x}_{1}\right)=$ $\bar{x}_{2}$ and $f_{R}\left(\bar{x}_{2}\right)=\bar{x}_{1}$ is given by

$$
\bar{x}_{1}=-\frac{s_{R} m_{L}+m_{R}}{s_{L} s_{R}-1}<\widetilde{P}_{-}^{*}, \quad \bar{x}_{2}=-\frac{s_{L} m_{R}+m_{L}}{s_{L} s_{R}-1}>\widetilde{P}_{+}^{*}
$$

Since when $s_{L} s_{R}>1$ and the unstable 2-cycle exists the second iterate $T^{2}$ increases in the two unstable fixed points $\bar{x}_{1}$ and $\bar{x}_{2}$, it follows that points above $\bar{x}_{1}$ are mapped above it and points below $\bar{x}_{2}$ are mapped below it, so that interval $J=\left[\bar{x}_{1}, \bar{x}_{2}\right]$ may be an invariant interval from which the trajectories cannot escape. This occurs when all the jumps are between the points of the 2-cycle. Noticing that $f_{L}\left(m_{L}\right)=\bar{x}_{2}$ iff $m_{L}=\bar{x}_{1}$ (and similarly $f_{R}\left(m_{R}\right)=\bar{x}_{1}$ iff $m_{R}=\bar{x}_{2}$ ), we can state the following:

Property 3. Let $s_{L} s_{R}>1$. Then $J=\left[\bar{x}_{1}, \bar{x}_{2}\right]$ is an invariant interval

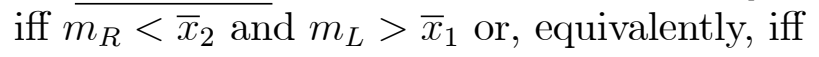

$$
m_{R}<-\frac{s_{L} m_{R}+m_{L}}{s_{L} s_{R}-1} \text { and } m_{L}>-\frac{s_{R} m_{L}+m_{R}}{s_{L} s_{R}-1} .
$$

\section{Subcase IV-A: Both fixed points stable.}

A first result of this subcase $\left(-1<s_{R, L}<0\right)$ is that no divergent trajectories can exist. A second result is that we have a coexistence of two locally stable fixed points. Thus, starting from any i.c., the system will converge to either $\widetilde{P}_{-}^{*}$ or $\widetilde{P}_{+}^{*}$. The basins of attraction of the two fixed points are made up of the union of alternating intervals. To show this, let us consider the immediate basin of each point and its preimages of any rank. The immediate basin of the fixed point $\widetilde{P}_{+}^{*}$ is given by interval $] 0, f_{R}^{-1}(0)[=] 0, \frac{-m_{R}}{s_{R}}$, and the trajectory of any i.c. in this interval converges to the stable fixed point on the right, without any negative value. Similarly, the immediate basin of the fixed point on the left is given by interval $] f_{L}^{-1}(0), 0[=] \frac{-m_{L}}{s_{L}}, 0[$. Then the global basins are made up of these intervals and all their preimages of any rank. Formally:

$$
\mathcal{B}\left(\widetilde{P}_{+}^{*}\right)=\cup_{n \geq 0} T^{-n}(] 0, \frac{-m_{R}}{s_{R}}[), \quad \mathcal{B}\left(\widetilde{P}_{-}^{*}\right)=\cup_{n \geq 0} T^{-n}(] \frac{-m_{L}}{s_{L}}, 0[)
$$

Note that the intervals belonging to the global basins of attraction are bounded (as for the immediate basins) by the preimages of the origin of any rank, that is: the stable set of the origin $W^{S}(0)$ is exactly the frontier of the two different basins, and (from Property 1 given above) the intervals are alternating on the real line. 


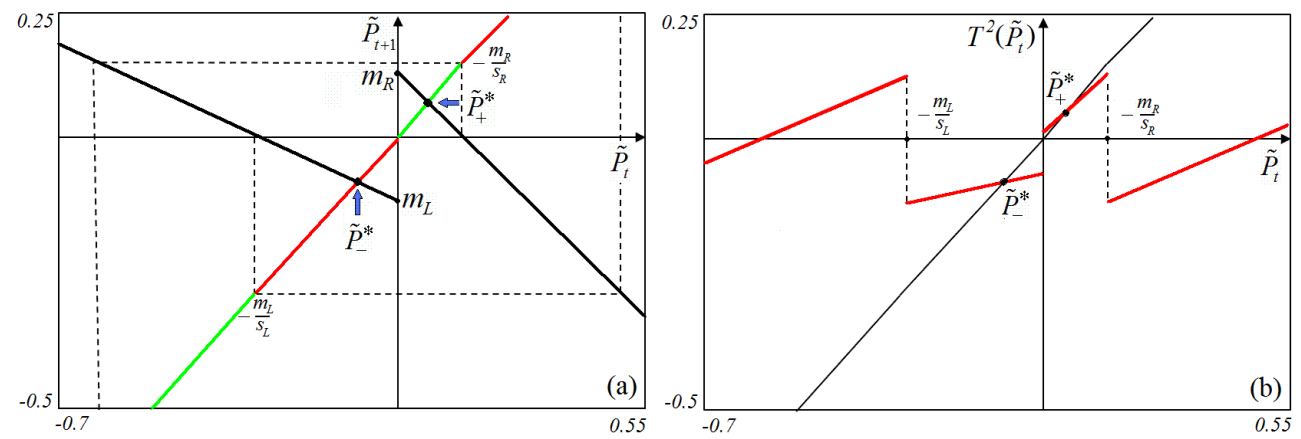

Fig. 8. Shape of map $T$ in (a) and of the second iterate $T^{2}$ in (b).

As an example, Fig. 8(a) shows the basins of attraction of the fixed points for the following set of parameters: $F=0 ; a=1 ; c^{1, a}=c^{1, b}=0.5$; $c^{2, a}=c^{2, b}=0.2 ; f^{2, a}=f^{2, b}=0.07 ; f^{1, a}=1.92 ; f^{1, b}=2.36$.

The dynamics changes from case IV-A to case IV-B when the slope of one branch is decreased and crosses the value -1 . This is a local bifurcation for the fixed point involved and, due to the linear component, is not a classical flip bifurcation. That is, when fixed points $\widetilde{P}_{+}^{*}$ and $\widetilde{P}_{-}^{*}$ become unstable it is not true that a stable 2-cycle will be created after the bifurcation. For this reason, we call it a degenerate flip bifurcation and, as we shall see, the result of such a degenerate flip bifurcation is not unique, and depends on the global behavior of the map (i.e. it also depends on the value of the other slope). Starting from parameters in the situation of stability for both fixed points, let us assume that $s_{R}=$ -1 is attained. Then at this bifurcation value the immediate basin of fixed point $\widetilde{P}_{+}^{*}$, that is interval $] 0, f_{R}^{-1}(0)[=] 0, \frac{-m_{R}}{s_{R}}[=] 0, m_{R}[$, is filled with stable (but not attracting) cycles of period 2 (in fact $T^{2}(x)=f_{R}^{2}(x)=x$ in this interval), and all points of the previous global basin of $\widetilde{P}_{+}^{*}$ are mapped into one such 2-cycle. After the bifurcation, when $s_{R}<-1$, only one fixed point is left. We can reason in the same way if $s_{L}=-1$ occurs first. Now let us consider the dynamics in this second regime for the slopes.

\section{Subcase IV-B: Only one fixed point stable.}

To fix the reasoning, let us assume the subcase in which $s_{R}<-1$ and $-1<s_{L}<0$ (as the other case in which $s_{L}<-1$ and $-1<s_{R}<0$ can be obtained immediately by exchanging $L$ and $R$ in all that follows and some related obvious changes). Clearly, this situation differs from the previous subcase in that we have a unique stable fixed point. As long as the second iterate of the map (map $T^{2}$ ) has only such cycles, that is, from Property 2, as long as $s_{L} s_{R}<1$, the unique stable fixed point $\widetilde{P}_{-}^{*}$ is almost globally attracting, as it attracts all the points different to 
unstable fixed point $\widetilde{P}_{+}^{*}, \widetilde{P}=0$ and the points which are mapped into them (constituting the stable sets $W^{S}\left(\widetilde{P}_{+}^{*}\right)$ and $\left.W^{S}(0)\right)$. Two numerical examples are shown in Fig. 9.

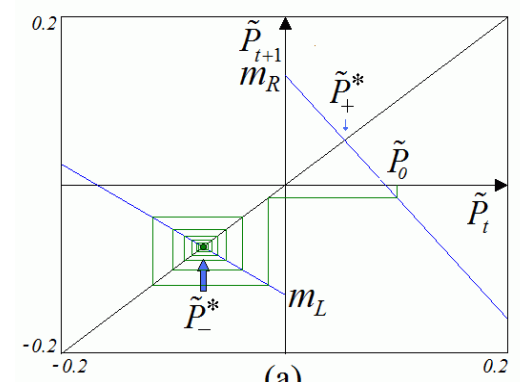

(a)

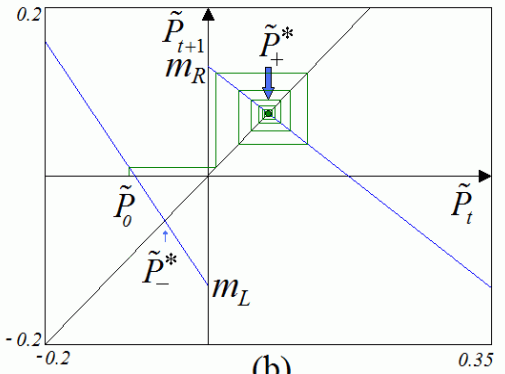

(b)

Fig. 9 (a) is obtained using $F=0 ; a=1 ; c^{1, a}=c^{1, b}=0.5$;

$$
c^{2, a}=c^{2, b}=0.2 ; f^{2, a}=f^{2, b}=0.07 ; f^{1, a}=2.225 ; f^{1, b}=2.9 \text {. (b) differs }
$$
from Fig. 9(a) for $f^{1, a}=2.9$ and $f^{1, b}=2.204$.

See also the qualitative picture in Fig. 10(a). The dynamics change when $s_{L} s_{R}>1$ and the unstable 2-cycle exists, with periodic points $\left\{\bar{x}_{1}, \bar{x}_{2}\right\}$. Then all the i.c. $\widetilde{P}<\bar{x}_{1}$ and $\widetilde{P}>\bar{x}_{2}$ have divergent trajectories, so that basin $\mathcal{B}(\infty)$ certainly includes set $]-\infty, \bar{x}_{1}[\cup] \bar{x}_{2},+\infty[$, while basin $\mathcal{B}\left(\widetilde{P}_{-}^{*}\right)$ belongs to the interval $J=\left[\bar{x}_{1}, \bar{x}_{2}\right]$ and is given in (17): by the immediate basin $] f_{L}^{-1}(0), 0[=] \frac{-m_{L}}{s_{L}}, 0[$ and then by all its preimages $\mathcal{B}\left(\widetilde{P}_{-}^{*}\right)=\cup_{n \geq 0} T^{-n}(] \frac{-m_{L}}{s_{L}}, 0[)$, and notice that now the frontier of $\mathcal{B}\left(\widetilde{P}_{-}^{*}\right)$ belongs to the stable set of the origin $W^{S}(0)$ (and is not equal to $W^{S}(0)$ because this stable set also includes other points: $-m_{R} / s_{R}$ and its preimages of any rank).

Moreover, as long as $J$ is invariant (see Property 3 ) then the closure of the basin is the whole interval bounded by the 2-cycle: $C_{l}\left(\mathcal{B}\left(\widetilde{P}_{-}^{*}\right)\right)=J$. That is, basin $\mathcal{B}\left(\widetilde{P}_{-}^{*}\right)$ is given by the points of $J$ after having eliminated the unstable cycles included therein and the related stable sets. In Fig. 10(b) we show the shape of map $T$ and its second iterate $T^{2}$. It is clear that of the four branches in the second iterate $T^{2}$, only one (corresponding to the immediate basin of $\widetilde{P}_{-}^{*}$ ) has a stable slope (between -1 and 0 ), while from $s_{L} s_{R}>1$ it follows that all other slopes are higher than 1. A similar pattern occurs for all solutions of equation $T^{k}(\widetilde{P})=\widetilde{P}$ for any $k$ : all branches of the $k$-th iterate $T^{k}$ are in modulus higher that 1 , except for the branch corresponding to the immediate basin of $\widetilde{P}_{-}^{*}$. Thus besides the unstable 2-cycle, all possible $k$-cycles are unstable. In fact, the following property (proof of which is given in the Appendix) holds: 

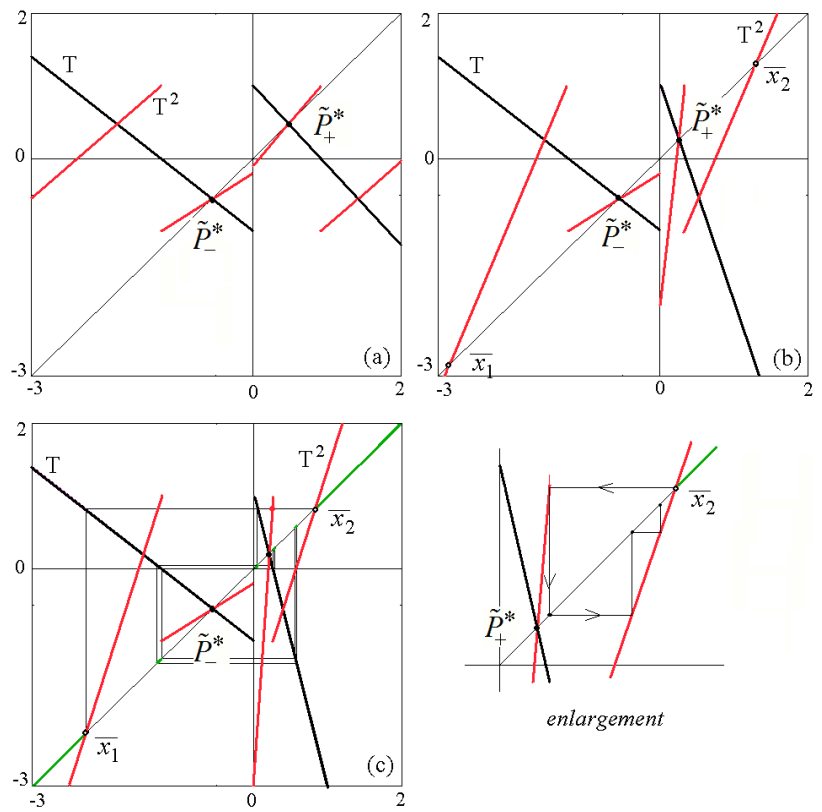

Fig. 10 Qualitative shape of map T in subcase IV-B, with $m_{L}=-1, m_{R}=1,-1<s_{L}=-0.8<0$ and $s_{R}<-1$. Then in (a) $s_{R}=-1.1\left(s_{L} s_{R}<1\right)$. In (b) $s_{R}=-3, s_{L} s_{R}>1$ and $I$ is invariant. In (c) $s_{R}=-4, s_{L} s_{R}>1$ and $I$ is not invariant. An enlargement of the region of (c) containing the homoclinic trajectory of the 2-cycle is presented in (d).

Property 4. Let $s_{L} s_{R}>1$. Then all the $k$-cycles are unstable.

A contact bifurcation (between the basin of divergent trajectories and basin $\left.\mathcal{B}\left(\widetilde{P}_{-}^{*}\right)\right)$ occurs when Property 3 no longer holds, and in this case, being $f_{L}\left(m_{L}\right)=m_{L}\left(1+s_{L}\right)<0$, bifurcation $f_{L}\left(m_{L}\right)=\bar{x}_{2}$ can never occur. Thus the only possibility is via bifurcation $m_{R}=\bar{x}_{2}$. Then, for $m_{R}>\bar{x}_{2}$ (i.e. $m_{R}>-\frac{s_{L} m_{R}+m_{L}}{s_{L} s_{R}-1}$ ) the points belonging to the interval ] $\left.\bar{x}_{2}, m_{R}\right]$ have divergent trajectories, and from Property 1 this interval has two distinct rank-1 preimages, which also belong to the basin of divergent trajectories, and in turn a sequence of infinitely many preimages. That is, for $m_{R}>\bar{x}_{2}$ basins $\mathcal{B}\left(\widetilde{P}_{-}^{*}\right)=\cup_{n \geq 0} T^{-n}(] \frac{-m_{L}}{s_{L}}, 0[)$ and $\left.\mathcal{B}(\infty)=]-\infty, \bar{x}_{1}[\cup] \bar{x}_{2},+\infty\left[\cup_{n \geq 0} T^{-n}(] \bar{x}_{2}, m_{R}\right]\right)$ inside $J$ consist of both infinitely many intervals which are intermingled in a complex way (see Fig. 10(c)). In fact, the preimages of any rank accumulate on the unstable periodic points existing there inside, which belong to a chaotic repellor $\Lambda$, which also includes the stable set $W^{S}(0)$. That is, the frontier separating the two basins $\mathcal{B}\left(\widetilde{P}_{-}^{*}\right)$ and $\mathcal{B}(\infty)$ is an invariant Cantor set $\Lambda$ of zero measure, on which the restriction of the mat $T$ is chaotic. The existence of chaotic dynamics is proven by fact that bifurcation $m_{R}=\bar{x}_{2}$ also corresponds to the homoclinic bifurcation of the 2-cycle. In fact, for the second iterate of map $T^{2}, \bar{x}_{2}$ is an unstable fixed point, 
and an homoclinic trajectory can be obtained taking the first preimage on its left and then infinitely many preimages on the right converging to $\bar{x}_{2}$ (such a homoclinic trajectory is shown in the enlargement of Fig. 10(c)), thus the proof is identical to that of Theorem 3(iii) given in the Appendix.

Subcase IV-C: Chaos $\left(s_{R, L}<-1\right)$.

Qualitative changes from the subcase IV-B described above can appear only if the remaining stable fixed point also undergoes a degenerate flip bifurcation. In this case, at the bifurcation value we still have the immediate basin filled with stable (but not attracting) 2-cycles, while after the bifurcation the dynamical result depends on the global properties, since it depends on both values of the two slopes. For example, if we decrease $s_{L}$ starting from a situation in which the closure is $C_{l}\left(\mathcal{B}\left(\widetilde{P}_{-}^{*}\right)\right)=J=\left[\bar{x}_{1}, \bar{x}_{2}\right]$ (as in Fig. 10(b)) (that is when $s_{L} s_{R}>1$ occurs as long as $m_{R}<\bar{x}_{2}$ and $m_{L}>\bar{x}_{1}$ ), we have bounded chaotic intervals. While if we decrease $s_{L}$ starting from a situation in which $\mathcal{B}\left(\widetilde{P}_{-}^{*}\right)$ and $\mathcal{B}(\infty)$ both exist inside $J=\left[\bar{x}_{1}, \bar{x}_{2}\right]$ (as in Fig. $10(\mathrm{c})$ ) (that is when $s_{L} s_{R}>1$ and either $m_{R}>\bar{x}_{2}$ or $m_{L}<\bar{x}_{1}$ ), then the result is that the generic trajectory is divergent, and the set of points which persists bounded is an invariant Cantor set $\Lambda$ of zero measure, and the restriction of the mat $T$ to $\Lambda$ is chaotic.

Now all existing cycles are necessarily unstable, since both slopes are less than -1 . So when the dynamics are bounded in the absorbing interval $J$ (from Property 3 as long as $m_{R}<\bar{x}_{2}$ and $m_{L}>\bar{x}_{1}$ ), we are faced with a chaotic regime. The reasoning is quite similar to that performed in the previous subsection (Case III), referred to the second iterate $T^{2}$ of the map. None of the trajectories can escape from the invariant interval $J=\left[\bar{x}_{1}, \bar{x}_{2}\right]$, infinitely many cycles exist, all unstable, and the Lyapunov exponent is necessarily positive. Thus the attractor is a set of $n$-cyclical chaotic intervals $(n \geq 1)$, and what may change is the number $n$ of components. As is well known, and as already mentioned in Case III, in piecewise-linear chaotic maps the chaotic intervals are bounded by the images of the critical point, which in our case are the jump at the discontinuity point: $f_{R}(0)=m_{R}$ and $f_{L}(0)=m_{L}$. And the bifurcations that cause the transition from the different situations are either border collision bifurcations or homoclinic bifurcations of repelling cycles outside the chaotic intervals, occurring due to the merging of the images of $m_{R}$ and $m_{L}$ with the unstable cycles. In particular, the homoclinic bifurcation of fixed point $\widetilde{P}_{-}^{*}$ can only occur when $f_{R}\left(m_{R}\right)=\widetilde{P}_{-}^{*}$, that is, when $m_{R}\left(1+s_{R}\right)=\frac{m_{L}}{1-s_{L}}$. Similarly, the homoclinic bifurcation of fixed point $\widetilde{P}_{+}^{*}$ can only occur when $f_{L}\left(m_{L}\right)=\widetilde{P}_{+}^{*}$, that is, when $m_{L}\left(1+s_{L}\right)=\frac{m_{R}}{1-s_{R}}$. Clearly the existence of bounded chaotic intervals (pure chaos which 
persist for intervals in the parameter values) is destroyed at the contact bifurcation of the chaotic intervals with the boundary of the invariant region $J=\left[\bar{x}_{1}, \bar{x}_{2}\right]$, which occurs when either $m_{R}=\bar{x}_{2}$ or $m_{L}=\bar{x}_{1}$ (after which, from Property $3, J=\left[\bar{x}_{1}, \bar{x}_{2}\right]$ is no longer invariant). As already mentioned above (in subcase IV-B), this situation corresponds to the homoclinic bifurcation of the 2-cycle, after which the generic trajectory will be divergent, except for a chaotic repellor $\Lambda$. In the enlargement of Fig. 10(c) we show a homoclinic trajectory of the 2-cycle when the final contact bifurcation occurs due to $m_{R}=\bar{x}_{2}$. Similarly, we can construct homoclinic trajectories in the case that $f_{L}\left(m_{L}\right)=\bar{x}_{2}$, i.e. $m_{L}=\bar{x}_{1}$. An examples is given in Fig. 11.

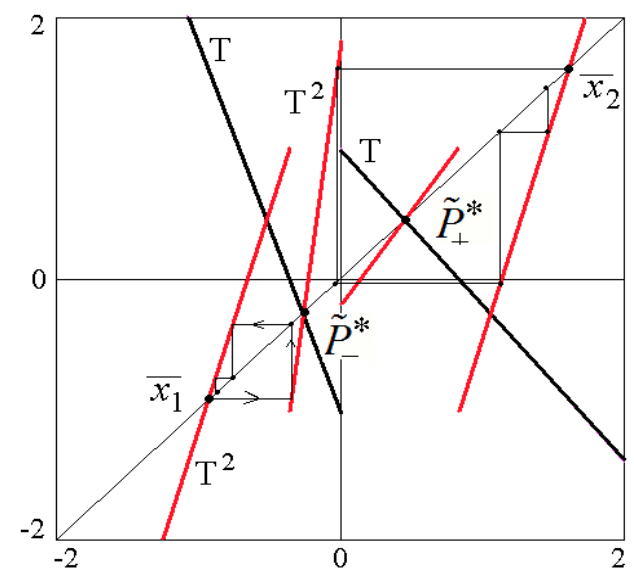

Fig. 11. Maps $T$ and $T^{2}$ at the following values of the parameters:

$$
s_{L}=-2.8, m_{L}=-1, s_{R}=-1.2, m_{R}=1 \text {. }
$$

We have proved the following

Theorem 4. Let $s_{R}<0, s_{L}<0, m_{R}>0, m_{L}<0$ in the map (9). Then

i) the two stable fixed points $\widetilde{P}_{-}^{*}$ and $\widetilde{P}_{+}^{*}$ coexist for $-1<s_{R, L}<0$; their basins (given in (17)) are alternating intervals separated by the the stable set of the origin $W^{S}(0)$;

ii) only one stable fixed point exists, almost globally attracting (except for the unstable fixed points and their preimages) for $s_{R}<-1$ and $-1<$ $s_{L}<0$, or $s_{L}<-1$ and $-1<s_{R}<0$, and $s_{L} s_{R}<1$;

iii) for $s_{R}<-1$ and $-1<s_{L}<0$, or $s_{L}<-1$ and $-1<s_{R}<0$; $s_{L} s_{R}>1, m_{R}<\bar{x}_{2}$ and $m_{L}>\bar{x}_{1}$, only one stable fixed point exists, and $C_{l}\left(B\left(\widetilde{P}^{*}\right)\right)=J=\left[\bar{x}_{1}, \bar{x}_{2}\right]$;

iv) for $s_{R}<-1$ and $-1<s_{L}<0$ or $s_{L}<-1$ and $-1<s_{R}<0$, $s_{L} s_{R}>1$, and $m_{R}>\bar{x}_{2}$ or $m_{L}<\bar{x}_{1}$, only one stable fixed point exists, and inside $J=\left[\bar{x}_{1}, \bar{x}_{2}\right]$, its basin $B\left(\widetilde{P}^{*}\right)$ is separated from $B(\infty)$ through a chaotic frontier $\Lambda$; 
v) for $s_{R, L}<-1, m_{R}<\bar{x}_{2}$ and $m_{L}>\bar{x}_{1}$, the attractor in $J=\left[\bar{x}_{1}, \bar{x}_{2}\right]$ consists of $n$-cyclical chaotic intervals with $n \geq 1$; the homoclinic bifurcation of the fixed points characterizing the merging of chaotic intervals occurs when $f_{R}\left(m_{R}\right)=\widetilde{P}_{-}^{*}$ (i.e. $\left.m_{R}\left(1+s_{R}\right)=\frac{m_{L}}{1-s_{L}}\right)$ and when $f_{L}\left(m_{L}\right)=\widetilde{P}_{+}^{*}$ (i.e. $\left.m_{L}\left(1+s_{L}\right)=\frac{m_{R}}{1-s_{R}}\right)$;

vi) for $s_{R, L}<-1$ and $m_{R}>\bar{x}_{2}$ or $m_{L}<\bar{x}_{1}$, no attractor exists: almost all of the trajectories are divergent, except for a chaotic repellor $\Lambda$ belonging to $J=\left[\bar{x}_{1}, \bar{x}_{2}\right]$

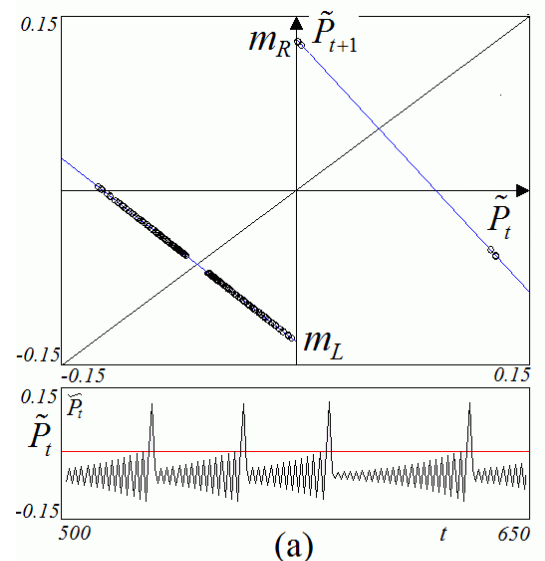

(a)

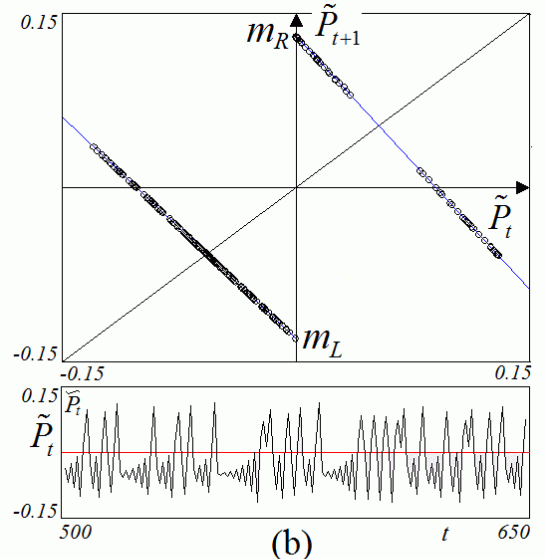

(b)
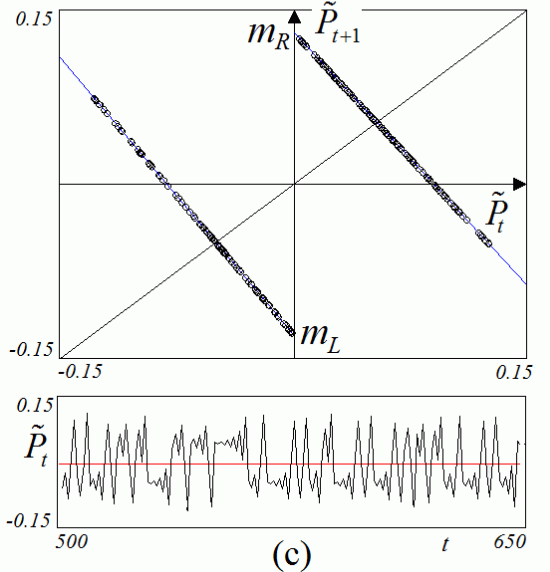

(c)

Fig. 12 The set of parameters used is $F=0 ; a=1 ; c^{1, a}=c^{1, b}=0.5$; $c^{2, a}=c^{2, b}=0.2 ; f^{2, a}=f^{2, b}=0.07 ; f^{1, b}=2.9$. The bifurcation parameter $f^{1, a}$ takes the values 2.55 (panel a), 2.72 (panel b) and 3.05 (panel c), corresponding to slopes $s_{L}=-1,05, s_{L}=-1.22$ and $s_{L}=-1.55$, respectively.

As an example, let us consider the case represented in Fig. 12, obtained by keeping the following set of parameters fixed: $F=0 ; a=1$; $c^{1, a}=c^{1, b}=0.5 ; c^{2, a}=c^{2, b}=0.2 ; f^{2, a}=f^{2, b}=0.07 ; f^{1, b}=2.9$ and letting parameter $f^{1, a}$ vary. Immediately after the degenerate flip bifurcation of the fixed point $\widetilde{P}_{-}^{*}$ (which becomes unstable) a three-bands 
chaotic attractor arises, shown in Fig. 12(a) $\left(f^{1, a}=2.55\right)$. Increasing the value of the parameter, the chaotic bands turn into two (Fig. 12(b), $\left.f^{1, a}=2.72\right)$ and then only one (Fig. 12(c), $\left.f^{1, a}=3.05\right)$. The scenarios shown in Fig. 12, when we decrease the slope of one branch, keeping the other slope fixed at a value lower than -1 , are cyclical chaotic intervals bounded by $m_{L}, m_{R}$, and their iterates. The transition from a qualitative case to the other occurs via a global bifurcation, the homoclinic bifurcation of one unstable fixed point, and this transition can be seen in the bifurcation diagram of Fig. 13. As slope $s_{L}$ is decreased, we can see the homoclinic bifurcations changing the number of chaotic bands. At $f^{1, a}=2.5$ the slope is $s_{L}=-1$ and fixed point $\widetilde{P}_{-}^{*}$ becomes unstable. There are three chaotic bands, until two of them merge in correspondence to the homoclinic bifurcation of $\widetilde{P}_{-}^{*}\left(f^{1, a}=h_{-} \simeq 2.62\right)$. When the homoclinic bifurcation of $\widetilde{P}_{+}^{*}$ occurs (at $f^{1, a}=h_{+}$), the two bands merge into a unique chaotic interval $\left[m_{L}, m_{R}\right]$, up to a value after which $f_{L}\left(m_{L}\right)>m_{R}$ and the chaotic interval is given by $\left[m_{L}, f_{L}\left(m_{L}\right)\right]$ up to the final contact bifurcation, occurring when $f_{L}\left(m_{L}\right)$ merges with point $\bar{x}_{2}$ of the unstable 2-cycle, after which the generic trajectory is divergent.

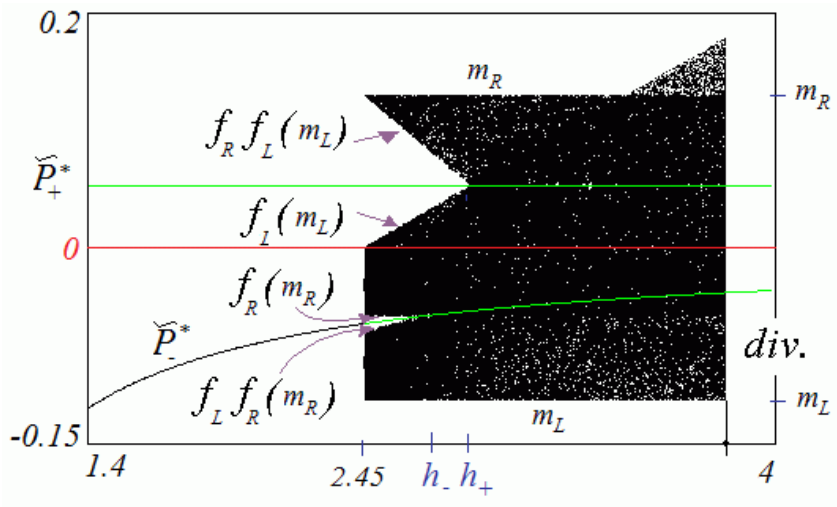

Fig. 13 One-dimensional bifurcation diagram for parameter $f^{1, a}$.

The time series plots of Fig. 12 show that passing from three to two and finally to only one chaotic band, switching between bull and bear price values becomes increasingly unpredictable.

Hence, this scenario is not only able to generate endogenous price fluctuations and thus replicates the phenomenon of excess volatility observed in many financial markets. It can also produce (persistent) periods of over- and undervaluation. Such boom-bust cycles are also a typical and recurrent phenomenon witnessed in many financial markets. From a policy perspective it is obviously quite important to understand what drives such dynamics. Highly volatile and distorted prices imply considerable market risks and are thus surely not beneficial for the welfare of an economy. We would like to stress that our paper reveals that 
such dynamics may be caused by (even one-dimensional) discontinuous maps, which should clearly illustrate their relevance ${ }^{9}$. Moreover, such discontinuous maps may originate from the behavior of boundedly rational agents who follow simple, yet plausible and empirically observable trading rules.

\section{Conclusion}

What drives the dynamics of financial markets? Since prices adjust with respect to demand and supply, a number of interesting models have been proposed in the recent past, which explicitly studied how agents determine their speculative investment positions. These papers have a strong empirical foundation since their main building blocks are supported by questionnaire and laboratory evidence according to which speculators rely on both technical and fundamental analysis to predict the directions of future market movements. We contribute to this research field by developing a novel financial market model with four different types of technical and fundamental traders. In particular, we take into account that traders may react asymmetrically to bull and bear market situations and that they may either formulate their orders on the basis of linear trading rules or simply prefer to trade fixed amounts of assets. From an economic point of view, our main results may be summarized as follows:

- In (special) Case I we only assume the existence of type 1 chartists and type 1 fundamentalists. As a result, the price dynamics either converges towards the fundamental value or it explodes.

- (Special) Case II highlights the particular role of type 2 traders. We have here an example of an extremely simple financial market model with heterogeneous traders which is nevertheless able to generate periodic or quasi-periodic price dynamics, at least for a subset of the parameter space, and thus reveals one potential engine of excess volatility.

- In Case III type 1 and type 2 chartists and type 1 and type 2 fundamentalists are active. However, type 1 chartists dominate type 1 fundamentalists and type 2 fundamentalists dominate type 2 chartists. Compared to (special) Case II, bounded price dynamics now is always chaotic.

\footnotetext{
${ }^{9}$ Note also that the first bifurcation in figure 13 is quite interesting from an economic point of view. Even a tiny shift in one of the behavioral parameters of our model may yield a switch from fixed point dynamics to fluctuations with a strong amplitude.
} 
- Finally, in Case IV we explore parameter constellations in which type 1 fundamentalists (strongly) dominate type 1 chartist and type 2 chartist dominate type 2 fundamentalists. Also this setup has the potential to generate chaotic price dynamics (but, for instance, also coexisting locally stable fixed points). Interestingly, we now observe intricate bull and bear market dynamics: asset prices may circle in the bull market for some time but then the market crashes and is characterized by temporary bear market fluctuations. Such erratic bull and bear market dynamics have first been studied by Day and Huang (1990), yet within a different model environment.

We would like to stress that our setup, i.e. the assumed set of trading rules, are quite natural extensions of what has been explored so far. In this sense, we add a new view to the literature which will hopefully help us to penetrate the complicated dynamics of financial markets even further. Let us finally point out a few avenues for future research. First, it would be interesting to explore a model in which the trading rules do not only take current prices into account. For instance, a technical trading rule in which orders depend on the most recent observed price trend can be assumed. Then the model would result in a two-dimensional discontinuous dynamical system. Second, the focus could be placed on more general trading rules which determine orders on the basis of a linear function but where the absolute size of the orders is also bounded. Finally, one may try to calibrate such models so that they are able to mimic certain stylized facts of financial markets. One way may be to add random shocks to the model, and we refer interest readers to a related paper by Westerhoff and Franke (2009). Another way may be to add (many) more rules to the market, as in Farmer and Joshi (2002).

\section{Appendix}

Proof of Theorem 1.

Due to the linearity of the map on both sides, when $s_{R}>1$ all trajectories on the $R$ side are divergent to $+\infty$, while trajectories on the $L$ side for $s_{L}>1$ are divergent to $-\infty$; for $0<s_{L}<1$ they are convergent to $\widetilde{P}_{t}=0$; for $s_{R}<0$ they are mapped on the right and then diverge to $+\infty$. When $0<s_{R}<1$, all trajectories on the $R$ side are convergent to $\widetilde{P}_{t}=0$, while the trajectories on the $L$ side for $s_{L}>1$ are divergent to $-\infty$; for $0<s_{L}<1$ they are convergent to $\widetilde{P}_{t}=0$; for $s_{R}<0$ they are mapped in the right side and then convergent to $\widetilde{P}_{t}=0$. When $s_{R}<0$, any point on the $R$ side is mapped on the $L$ side in one iteration and for $s_{L}>1$ all trajectories are divergent to $-\infty$; for $0<s_{L}<1$ all trajectories are convergent to $\widetilde{P}_{t}=0$ from the $L$ side; for 
$s_{L}<0$ the trajectories are convergent to $\widetilde{P}_{t}=0$ with oscillations as long as $s_{R} s_{L}<1$ (the second iterate of the map has a stable fixed point), otherwise all the trajectories diverge with oscillations. The particular cases in which the slopes amount to 0 or \pm 1 are immediate.

Proof of Theorem 2.

Looking for $(n+1)$-periodic orbits with one point on the $L$ side and $n$ on the $R$ side, we have to consider the fixed points of the iterated map

$$
f_{R}^{n} \circ f_{L}\left(\widetilde{P}_{t}\right)=\widetilde{P}_{t}+m_{L}+n m_{R}
$$

When we have $1 m_{L}+n m_{R}=0, f_{R}^{n} \circ f_{L}\left(\widetilde{P}_{t}\right)=\widetilde{P}_{t}$, and inside $I$ all points are periodic of period $n+1$, in which case the points outside $I$ become periodic in a finite number of steps (also called eventually periodic or preperiodic). In general, given a periodic orbit with $k$ distinct points on the $L$ side and $n$ distinct points on the $R$ side, we have

$$
f^{n+k}\left(\widetilde{P}_{t}\right)=f_{R}^{n} \circ f_{L}^{k}\left(\widetilde{P}_{t}\right)=\widetilde{P}_{t}+k m_{L}+n m_{R}
$$

Thus all of the points are of period $(n+k)$ inside $I$ (and eventually periodic outside $I$ ) when $k m_{L}+n m_{R}=0$, or, equivalently $m_{L}=-\frac{n}{k} m_{R}$. So we have to consider the rational numbers ,

$$
\frac{m_{L}}{-m_{R}}=\frac{n}{k}
$$

where $n \geq 1$ and $k \geq 1$ are integers. This means that if $m_{L}=-\rho m_{R}$, where $\rho=\frac{m_{L}}{-m_{R}}=\frac{n}{k}$ is rational, all orbits in $I$ are periodic, while if $m_{L}=-\rho m_{R}$, where $\rho$ is irrational all orbits in $I$ are quasiperiodic. Thus considering $m_{L}=-\rho m_{R}$, the absorbing interval $I$ is filled with periodic orbits when $\rho$ is rational or quasiperiodic orbits when $\rho$ is irrational. Moreover, when $\rho$ is rational, defining $\rho=\frac{m_{L}}{-m_{R}}=\frac{n}{k}$, where $n$ and $k$ are both positive integers with no common divisors, all orbits have period $(n+k)$, where $k$ is the number of periodic points on the $L$ side and $n$ is the number of periodic points on the $R$ side.

Proof of Theorem 3.

What is left to prove is point (iii) of the Theorem. Let us consider the contact bifurcation that occurs when $m_{L}=\widetilde{P}_{+}^{*}\left(=\frac{m_{R}}{1-s_{R}}\right)$ and that leads to a homoclinic fixed point after the bifurcation (the reasoning is similar in the other case). Then the existence of homoclinic orbits to the fixed point is enough for the existence of chaos on some invariant Cantor set $\Lambda$. There are several proofs of this. Here we propose a simple proof. Let us consider a neighborhood $U$ of $\widetilde{P}_{+}^{*}$ for $m_{L}>\frac{m_{R}}{1-s_{R}}$. By using the two inverses $f_{R}^{-1}(z)=\frac{z-m_{R}}{s_{R}}$, which gives points on the $R$ 
side, and $f_{L}^{-1}(z)=\frac{z-m_{L}}{s_{L}}$, which gives points on the $L$ side, consider its two distinct rank-1 preimage: $f_{R}^{-1}(U)$ and $f_{L}^{-1}(U)$ are on opposite sides with respect to the origin, and thus disjoint. Then let us consider their successive preimages using the $f_{R}^{-1}$ for $N$ iterations, as long as $\left(f_{R}^{-1}\right)^{N_{0}}$ $f_{L}^{-1}(U) \subset U$. Then define $U_{1}=\left(f_{R}^{-1}\right)^{N} \circ f_{L}^{-1}(U)$ and $U_{0}=\left(f_{R}^{-1}\right)^{N+1}(U)$, which are clearly disjoint and inside $U$. Thus, by construction, we have that $T^{N+1}\left(U_{1}\right)$ covers (includes) $U_{0} \cup U_{1}$ and $T^{N+1}\left(U_{0}\right)$ covers $U_{0} \cup U_{1}$. This is enough to state that inside $U$ there exist an invariant Cantor set $\Lambda$ on which map $T^{N+1}$ is conjugated with the shift map, on which it is chaotic, and thus also $T$ is chaotic (see, e.g. Devaney, 1986, Gardini 1994, Gardini et al. 2009).

\section{Proof of Property 4.}

The statement is obvious if $s_{L}<-1$ and $s_{R}<-1$. Thus we are only interested in case (a) $-1<s_{L}<0$ and $s_{R}<-1$ or (b) $-1<s_{R}<0$ and $s_{L}<-1$. Let us fix the reasoning in case (a). The other case is similar with obvious changes. If $-1<s_{L}<0$, fixed point $\widetilde{P}_{-}^{*}$ is stable, and its immediate basin of attraction is given by the interval $] f_{L}^{-1}(0), 0[$. It follows that all existing $k$-cycles, which must belong to the interval $J=\left[\bar{x}_{1}, \bar{x}_{2}\right]$, must also have periodic points in the interval $] \bar{x}_{1}, f_{L}^{-1}(0)[$, and thus such a periodic point in the $L$ region is mapped by $T$ in the $R$ region. This means that the sequence of symbols associated with a $k$ cycle is necessarily constituted by letters such as $(L R) \ldots R \ldots(L R) \ldots R \ldots$ (i.e. two consecutive $L_{\mathrm{s}}$ cannot occur) and the related eigenvalue is $s_{k}=\left(s_{L} s_{R}\right) \ldots s_{R} \ldots\left(s_{L} s_{R}\right) \ldots s_{R} \ldots$ Thus $\left(s_{L} s_{R}\right)>1$ and $s_{R}<-1$ imply $\left|s_{k}\right|>1$.

\section{References}

Avrutin, V., Schanz, M., 2006. Multi-parametric bifurcations in a scalar piecewise-linear map. Nonlinearity 19, 531-552.

Avrutin, V., Schanz, M., 2008. On the fully developed bandcount adding scenario. Nonlinearity 21, 1077-1103.

Avrutin, V., Schanz, M., Banerjee, S., 2006. Multi-parametric bifurcations in a piecewise-linear discontinuous map. Nonlinearity 19, 18751906.

Avrutin, V., Eckstein, B., Schanz, M., 2008a. The bandcount increment scenario. I. Basic Structures. Proceedings of the Royal Society A: Mathematical, Physical and Engineering Sciences 464, 1867-1883.

Avrutin, V., Eckstein, B., Schanz, M., 2008b. The bandcount increment scenario. II. Interior Structures. Proceedings of the Royal Society A: Mathematical, Physical and Engineering Sciences 464, 2247-2263.

Avrutin, V., Eckstein, B., Schanz, M., 2009. The bandcount increment scenario. III. Deformed Structures. Proceedings of the Royal 
Society A: Mathematical, Physical and Engineering Sciences 465, 41-57.

Banerjee, S., Karthik, M.S., Yuan, G., Yorke, J.A., 2000. Bifurcations in One-Dimensional Piecewise Smooth Maps - Theory and Applications in Switching Circuits. IEEE Transactions on Circuits and Systems I: Fundamental Theory and Applications 47, 389-394.

Beja, A., Goldman, M., 1980. On the dynamic behaviour of prices in disequilibrium. Journal of Finance 34, 235-247.

Böhm V., Kaas, L., 2000. Differential savings, factor shares and endogenous growth cycles. Journal of Economic Dynamics and Control 24, 965-980.

Boswijk, P., Hommes, C., Manzan, S., 2007. Behavioral heterogeneity in stock prices. Journal of Economic Dynamics and Control 31, 1938-1970.

Bouchaud, J.-P., Farmer, D., Lillo, F., 2009. How markets slowly digest changes in supply and demand. In: Hens, T., Schenk-Hoppé, K.R. (Eds). Handbook of Financial Markets: Dynamics and Evolution. Amsterdam: North-Holland, 57-160.

Brock, W., Hommes, C., 1998. Heterogeneous beliefs and routes to chaos in a simple asset pricing model. Journal of Economic Dynamics Control 22, 1235-1274.

Chen, S.-H., Chang, C.L., Du, Y.-R., 2009. Agent-based economic models and econometrics. Knowledge Engineering Review, in press.

Chiarella, C., 1992. The dynamics of speculative behavior. Annals of Operations Research 37, 101-123.

Chiarella, C., Dieci, R., Gardini, L., 2002. Speculative behaviour and complex asset price dynamics: A global analysis. Journal of Economic Behavior and Organization 49, 173-197.

Chiarella, C., Dieci, R., Gardini, L., 2005. The dynamic interaction of speculation and diversification. Applied Mathematical Finance 12, $17-52$.

Chiarella, C., Dieci, R., He, X.-Z., 2009. Heterogeneity, market mechanisms, and asset price dynamics. In: Hens, T., Schenk-Hoppé, K.R. (Eds.). Handbook of Financial Markets: Dynamics and Evolution. Amsterdam: North-Holland, 277-344.

Day, R., 1982. Irregular growth cycles. American Economic Review 72, 406-414.

Day, R., 1994. Complex Economic Dynamics. Cambridge: MIT Press.

Day, R., Pianigiani, G., 1991. Statistical dynamics and economics. Journal of Economic Behavior and Organanization 16, 37-83.

Day, R., Shafer, W., 1987. Ergodic fluctuationsin deterministic economic models. Journal of Economic Behavior and Organanization 8, 
339-361.

Day, R., Huang, W., 1990. Bulls, bears and market sheep. Journal of Economic Behavior and Organization 14, 299-329.

Devaney, R., 1986. An Introduction to Chaotic Dynamical Systems. Menlo Park, California: The Benjamin/Cummings Publishing Co..

Di Bernardo, M., Budd, C.J., Champneys, A.R., Kowalczyk, P., 2008. Piecewise-smooth Dynamical Systems, Theory and Applications. New York: Springer Verlag.

Farmer, D., Joshi, S., 2002. The price dynamics of common trading strategies. Journal of Economic Behavior and Organization 49, 149-171.

Ferri, P., Greenberg, E., Day, R., 2001. The Phillips curve, regime switching and the NAIRU. Journal of Economic Behavior and Organization 46, 23-37.

Fournier-Prunaret, D., Mira, C., Gardini, L., 1997. Some contact bifurcations in two-dimensional examples. Grazer Mathematische Berichte $334,77-96$.

Franke, R., 2009. On the specification of noise in two agent-based asset pricing models. Working Paper, University of Kiel.

Franke, R., Westerhoff, F., 2009. Validation of a structural stochastic volatility model of asset pricing. Working Paper, University of Kiel.

Gardini, L., 1994. Homoclinic bifurcations in n-dimensional endomorphisms, due to expanding periodic points. Nonlinear Analysis, Theory, Methods \& Applications 23, 1039-1089.

Gardini, L., Hommes, C., Tramontana, F., de Vilder, R., 2009. Forward and Backward Dnamics in implicitly defined Overlapping Generations Models. Journal of Economic Behavior and Organization 71, 110-129.

Gaunersdorfer, A., Hommes, C., 2007. A nonlinear structural model for volatility clustering. In: Teyssière, G., Kirman, A. (Eds.). Long memory in economics. Berlin: Springer, 265-288.

Graham, B., Dodd, D., 1951. Security Analysis. New York: McGraw Hill.

He, X.-Z., Li, Y., 2008. Heterogeneity, convergence and autocorrelations. Quantitative Finance 8, 58-79.

He, X.-Z., Westerhoff, F., 2005. Commodity markets, price limiters and speculative price dynamics. Journal of Economic Dynamics and Control 29, 1577-1596.

Hommes, C.H., 1991. Chaotic dynamics in economic models. Ph.D. Thesis. Groningen: Wolters-Noodhoff.

Hommes, C.H., Nusse, H., 1991. Period three to period two bifurcations for piecewise linear models. Journal of Economics 54, 157-169. 
Hommes, C.H., Nusse, H., Simonovits, A., 1995. Cycles and chaos in a socialist economy. Journal of Economic Dynamics and Control 19, $155-179$.

Hommes, C.H., 1995. A reconsideration of Hick's non-linear trade cycle model. Structural Change and Economic Dynamics 6, 435-459.

Hommes, C.H., Sonnemans, J., Tuinstra, J., van de Velden, H., 2005. Coordination of expectations in asset pricing experiments. Review of Financial Studies 18, 955-980.

Hommes, C.H., Wagener, F., 2009. Complex evolutionary systems in behavioral finance. In: Hens, T., Schenk-Hoppé, K.R. (Eds.). Handbook of Financial Markets: Dynamics and Evolution. Amsterdam: NorthHolland, 217-276.

Jain, P., Banerjee, S., 2003. Border-collision bifurcations in onedimensional discontinuous maps. International Journal of Bifurcation and Chaos 13, 3341-3351.

Kirman, A., 1991. Epidemics of opinion and speculative bubbles in financial markets. In: Taylor, M. (Ed.). Money and Financial Markets. Oxford: Blackwell, 354-368.

LeBaron, B., 2006. Agent-based computational finance. In: Tesfatsion, L., Judd, K. (Eds.). Handbook of Computational Economics Vol. 2: Agent-based Computational Economics. Amsterdam, North-Holland, 1187-1233.

Lux, T., 1998. The socio-economic dynamics of speculative markets: Interacting agents, chaos, and the fat tails of return distributions. Journal of Economic Behavior and Organization 33, 143-165.

Lux, T., 2009a. Financial power laws: Empirical evidence, models and mechanisms. In. Cioffi-Revilla, C. (Ed.). Power laws in the social sciences: Discovering complexity and non-equilibrium dynamics in the social universe. Cambridge: Cambridge University Press, in press.

Lux, T., 2009b. Stochastic behavioural asset-pricing models and the stylize facts. In: Hens, T., Schenk-Hoppé, K.R. (Eds.). Handbook of Financial Markets: Dynamics and Evolution. Amsterdam: NorthHolland, 161-216.

Menkhoff, L., Taylor, M., 2007. The obstinate passion of foreign exchange professionals: technical analysis. Journal of Economic Literature 45, 936-972.

Metcaf, C.J., 2008. The dynamics of the Stiglitz policy in the RSS model. Chaos, Solitons \& Fractals 37, 652-661.

Mira, C., Gardini, L., Barugola, A., Cathala, J.C., 1996. Chaotic Dynamics in two-dimensional noninvertible maps. Singapore: World Scientific. 
Murphy, J., 1999. Technical Analysis of Financial Markets. New York, New York Institute of Finance.

Nusse, H.E., Yorke, J.A., 1992. Border-collision bifurcations including period two to period three for piecewise smooth systems. Physica D $57,39-57$.

Nusse, H.E., Yorke, J.A., 1995. Border-collision bifurcations for piecewise smooth one-dimensional maps. International Journal of Bifurcations and Chaos 5, 189-207.

Puu, T., Sushko, I., 2002. Oligopoly Dynamics, Models and Tools. New York: Springer Verlag.

Shiller, R., 2005. Irrational exuberance (2nd edition). Princeton: Princeton University Press.

Shiller, R., 2008. The subprime solution. Princeton: Princeton University Press.

Smith, V., Suchanek, G., Williams, A., 1988. Bubbles, crashes, and endogenous expectations in experimental spot asset markets. Econometrica $56,1119-1151$.

Sornette, D., 2003. Why stock markets crash. Princeton: Princeton University Press.

Tramontana, F., Gardini, L., Puu, T., 2009a. Duopoly games with alternative technologies. Journal of Economic Dynamics and Control 33, 250-265.

Tramontana, F., Gardini, L., Bischi, G.I., 2009b. Bifurcation curves in discontinuous maps. Discrete and Continuous Dynamical Systems, Serie B, in press.

Tramontana, F., Gardini, L., Ferri, P., 2009c. The dynamics of the NAIRU model with two switching regimes. Journal of Economic Dynamics and Control, in press.

Westerhoff, F., 2004. Multiasset market dynamics. Macroeconomic Dynamics 8, 596-616.

Westerhoff, F., Dieci, R., 2006. The effectiveness of Keynes-Tobin transaction taxes when heterogeneous agents can trade in different markets: a behavioral finance approach. Journal of Economic Dynamics and Control 30, 293-322.

Westerhoff, F., Franke, R., 2009. Converse trading strategies, intrinsic noise and the stylized facts of financial markets. Working Paper, University of Bamberg.

Westerhoff, F., 2009. Exchange rate dynamics: A nonlinear survey. In: Rosser, J.B., Jr. (Ed.). Handbook of Research on Complexity. Cheltenham: Edward Elgar.

Zhu, M., Chiarella, C., He, X.-Z., Wang, D., 2009. Does the market maker stabilize the market?. Physica A 388, 3164-3180. 